Int. J. Speleol. 10 (1978), pp. $351-379$

\title{
Spontane und induzierte Aktivitätsmuster bei troglobionten Käfern (Genera Aphaenops, Geotrechus, Speonomus)
}

\author{
von \\ G. LAMPRECHT ${ }^{(1)}$ und F. WEBER ${ }^{(2)}$
}

Spontaneous and induced activity patterns in troglobite beetles (Genera Aphaenops, Geotrechus, Speonomus)

\section{SUMMARY}

In constant temperature the troglobite beetles Aphaenops cerberus and pluto, Geotrechus orthe'us and Speonomus diecki are aperiodically active. By periodogram analysis neither circadian nor ultradian or infradian periodic components can be found. Moreover there are no relevant correlations between the lengths of successive activity bursts and rest pauses. Consequently the activity patterns are stochasticly structured. - In cases with constancy of activity respectively rest behaviour the probabilities for the transition from activity to rest and from rest to activity are computed using the frequency histograms of lengths of activity bursts and rest pauses. The transition probability is time-invariant if the observed histogram can be approximated to the negative exponential function $y=a \cdot e^{-f \cdot x}$. The transition probability increases continuously if the observed histogram can be approximated to the Poisson or normal function. $-74 \%$ of the investigated frequency histograms of the length of activity bursts and $57 \%$ of the histograms of the lengths of rest pauses can be approsimated to one of the tested functions. -Aphaenops and Geotrechus specimens do not react to changes of the illumination intensity. Conversely temperature cycles induce distinct activity periodicities. In Aphaenops the mean length of activity bursts is -on the average - less temperaturedependent than the mean length of rest pauses. Moreover, in this species the mean length of burst is weakly negatively correlated with the mean length of pauses. - The evolution and adaptiic reactions of the random mechanism of activity control in troglobite animals are discussed.

\section{FINI.EITUNG}

Die spontane Laufaktivität der meisten epigäisch aktiven Tierarten wird von cinem endogenen (selbsterregten) circadianen Oszillator gesteuert. Im Zuge der Evolution unter Höhlenbedingungen degeneriert die «innere Uhr», ohne

(1) 1)-28 Bremen, Rechen/entrum der Universität, Achterstraße

(2) 1)-4t Münster, /oologisches Institut der Universität, Badestr. 9 
dass gleichzeitig auch in auffälliger Weise die spontane Lokomotion reduziert wird (Besprechung der Literatur in Kap. 4.1.). Es stellt sich die Frage, welcher aktivitätsverteilende Mechanismus im Ablauf der regressiven Evolution an die Stelle des circadianen Oszillators tritt.

Lehmann, Neumann und Kaiser (1974) sowie Kaiser und Lehmann (1975) haben einen neuen Ansatz zur Analyse aperiodischer Aktivitätsmuster entwikkelt. Sie konnten bei Winkerkrabben die unter Konstantbedingungen registrierten aperiodischen Sequenzen aus Aktivitätsschüben und Ruhepausen mit Hilfe einfacher Zufalls-Gesetzmässigkeiten beschreiben: (1) die Länge der Aktivitätsschübe und die Länge der Ruhepausen ist unabhängig von der Länge der vorausgehenden Schübe bzw. Pausen; (2) die Wahrscheinlichkeit für den Übergang von Aktivität zu Ruhe ist zu jedem Zeitpunkt innerhalb der Aktivitätsschübe gleich gross; (3) die Wahrscheinlichkeit für den Übergang von Ruhe zu Aktivität ist entweder ebenfalls zeitinvariant oder verändert sich regelhaft mit der Dauer der Pausen.

Wir untersuchen in dieser Arbeit, ob die spontanen Aktivitätsmuster extrem evoluierter Höhlenkäfer (Arten der Carabidengattungen Aphaenops und Geotrechus sowie der Catopidengattung Speonomus) ebenfalls durch die Annahme ausschliesslich zufällig verteilter Übergänge von Aktivität zu Ruhe bzw. von Ruhe zu Aktivität beschrieben werden können. - Höhlentiere kommen nicht nur im Inneren von Höhlen vor, sondern unter geeigneten Bedingungen auch in Höhleneingangsbereichen, wo sie schwachen tages-und jahreszeitlichen Veränderungen des Lichtes und der Temperatur ausgesetzt sein können (Juberthie, 1969). Wir prüfen hier deshalb auch, ob und in welcher Weise die Muster der Spontanaktivität durch diese Umweltfaktoren beeinflusst werden.

\section{MATERIAL UND METHODE}

\subsection{Material}

Die untersuchten Arten (Aphaenops cerberus und A. pluto, Geotrechus orpheus und Speonomus diecki) sind blind und unpigmentiert. Die für Höhlentiere typischen anatomischen Merkmale sind bei den Aphaenops-Arten besonders extrem ausgebildet: der Körper ist schmal, Antennen und Beine sind verlängert, die fragile Kutikula ist mit langen Tastborsten besetzt (Vandel, 1965). Die Tiere stammen aus Höhlen des Département Ariège (Frankreich, Pyrenäen). Sie wurden. in Kühltaschen nach Münster (Westdeutschland) transportiert und hier in feuchten, mit Lehm ausgestrichenen Gipsschalen bei 8 bis $9^{\circ} \mathrm{C}$ im Dauerdunkel gehalten.

Ein-bis zweimal im Monat wurden sie mit zerschnittenen (irillen-Larven gefüttert. Die Käfer überlebten bis zu einem Jahr und wurden zum Teil mehr- 
mals in den Versuchen eingesetzt.

\subsection{Aktographen und Registrierung}

Die Aktographen, in denen die Tiere einzeln untersucht wurden, bestehen aus zwei gleich grossen Plastikschalen $(\varnothing 8,5 \mathrm{~cm})$. Auf die untere, mit Gips ausgefüllte Schale wird die obere umgekehrt aufgesetzt. In den Gipsboden ist die Öffnung einer Flasche eingelassen, die als Wasserreservoir dient und den Gipsboden feucht hält. Während der 16- bis 20- tägigen Experimente wurden die Tiere nicht gefüttert, um Störungen zu vermeiden.

Die Laufaktivität wird durch eine Infrarotlichtschranke $(800 \mathrm{~nm})$ erfasst. Die Anzahl der Passagen durch den Messtrahl wird elektronisch gezählt und alle 10 min auf ein Magnetband und einen Blattschreiber übertragen. Jedes Einzeltierexperiment liefert also eine lückenlose Serie von 2600 bis $300010 \mathrm{~min}$ Werten.

\subsection{Auswertung "}

Zur Ermittlung von periodischen Anteilen innerhalb einer Meßserie nach dem Periodogramm-Verfahren von Lamprecht $u$. Weber (1970) werden die aufeinanderfolgenden Meßwerte in Form einer Matrix angeordent:

\begin{tabular}{ccccccc}
\hline $\mathrm{A}_{1}$ & $\mathrm{~A}_{\mathrm{n}+1}$ & $\mathrm{~A}_{2 \mathrm{n}+1}$ & $*$ & $*$ & $*$ & $\mathrm{M}_{1 \pm \mathrm{s}_{1}}$ \\
\hline $\mathrm{A}_{2}$ & $\mathrm{~A}_{\mathrm{n}+2}$ & $\mathrm{~A}_{2 \mathrm{n}+2}$ & $*$ & $*$ & $*$ & $\mathrm{M}_{2 \pm \mathrm{s} 2}$ \\
\hline $\mathrm{A}_{3}$ & $\mathrm{~A}_{\mathrm{n}+3}$ & $\mathrm{~A}_{2 \mathrm{n}+3}$ & $*$ & $*$ & $*$ & $\mathrm{M}_{3} \pm \mathrm{s}_{3}$ \\
\hline$*$ & $*$ & $*$ & $*$ & $*$ & $\mathrm{~A}_{\mathrm{m}-1}$ & $*$ \\
\hline$*$ & $*$ & $*$ & $*$ & $*$ & $\mathrm{~A}_{\mathrm{m}}$ & $*$ \\
\hline$*$ & $*$ & $*$ & $*$ & $*$ & & $*$ \\
\hline $\mathrm{A}_{n}$ & $\mathrm{~A}_{2 \mathrm{n}}$ & $\mathrm{A}_{3 \mathrm{n}}$ & $*$ & $*$ & & $\mathrm{M}_{\mathrm{n}} \pm \mathrm{sn}$ \\
\hline
\end{tabular}

Dic Zeilenanzahl $\mathrm{n}$ wird schrittweise von 5 bis 250 variiert (das entspricht 50 min bis $42 \mathrm{Std}$ ). Es werden also von ein- und derselben Meßserie 246 verschiedene Matrizen gebildet. Für jede Zeile j einer Matrix werden Mittelwert $\mathbf{M}_{\mathrm{j}}$ und Streuung $s$, errechnet. Die Summe der Streuungen um die Zeilenmittel-

1) Durchgefuhrt an den Rechenanlagen der Universiäten Bremen und Münster. 


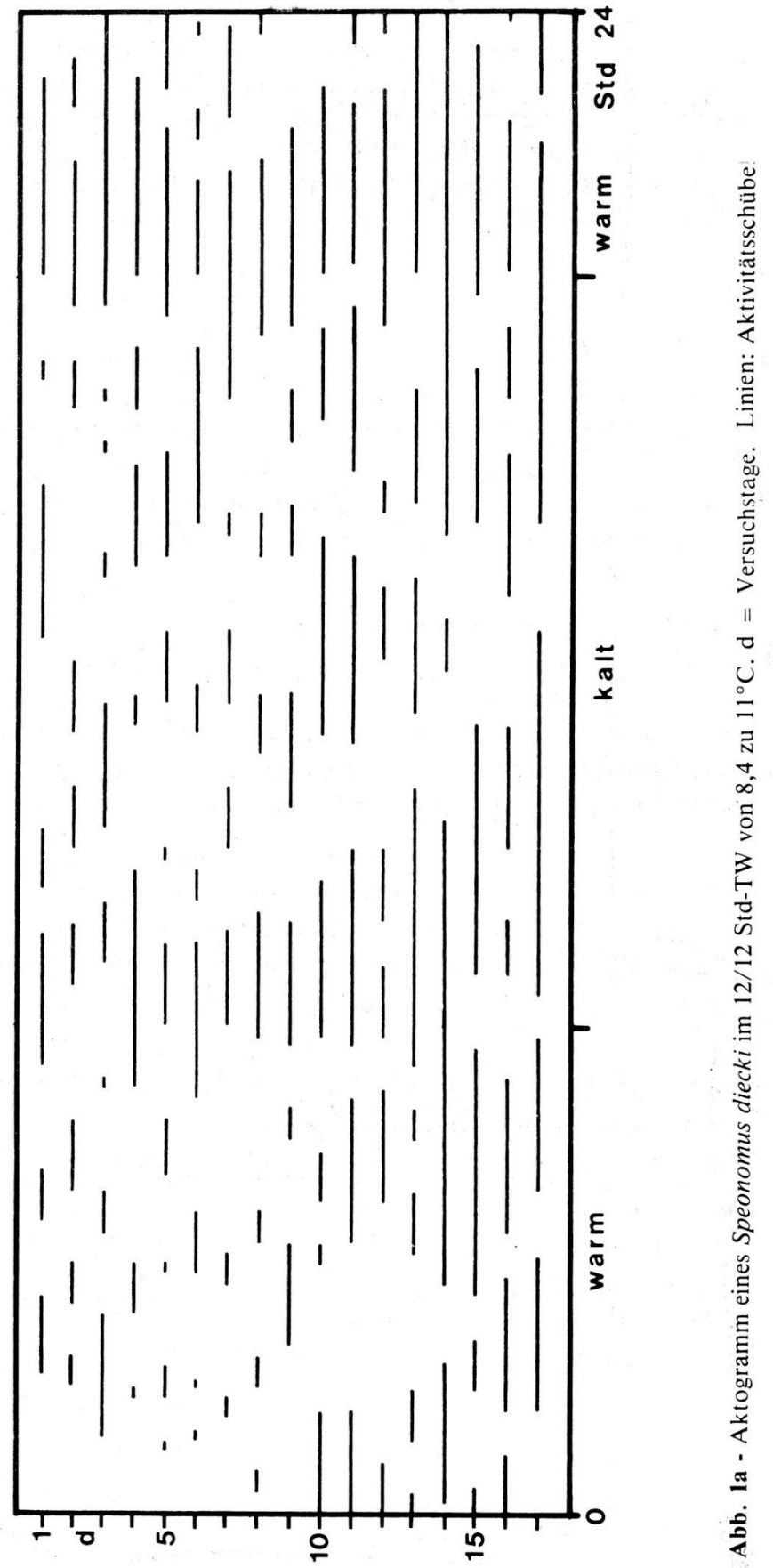




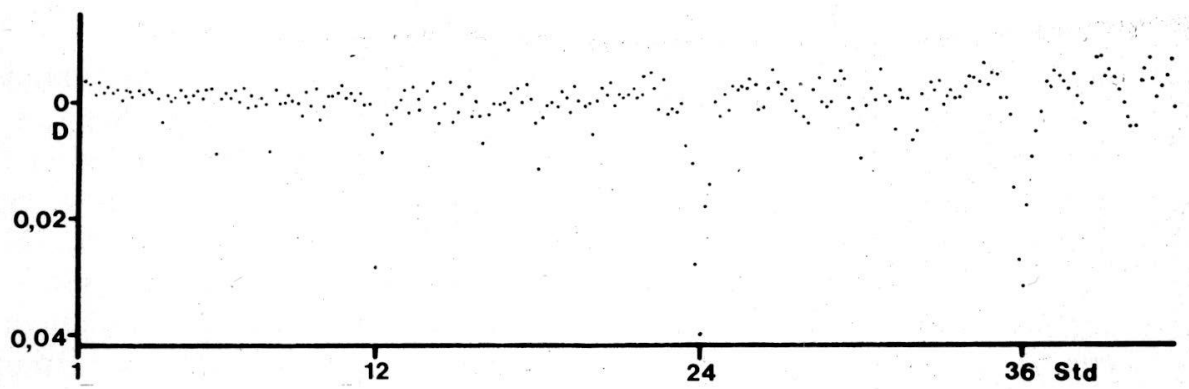

\begin{abstract}
Abb. Ib
Das Periodogramm des in Abb. 1a dargestellten Aktogramms im Bereich von 1 bis 42 Std. D (Ordinate): Abstand der Periodogrammwerte vom Mittel aller Periodogrammwerte. Das Periodogramm basiert auf den Origininalwerten, berücksichtigt also auch die Aktivitätsintensität. Bei 24 Std tritt ein tiefes Minimum auf. Das Nebenminimum bei $12 \mathrm{Std}$ ist eine Folge der Induktion von Aktivitätsschüben sowohl an der Grenze «warm - kalt» als auch an der Grenze «kalt - warm».
\end{abstract}

werte ist dann minimal, wenn phasengleiche Meßwerte eines periodisch aktiven Tieres in derselben Zeile eingeordnet sind. Näheres zum Verfahren, insbesondere zur Normierung der Matrix, siehe bei Lamprecht und Weber (1970). $\mathrm{Ob}$ und bei welchen Zeilenanzahlen Minima in der Summe der Streuungen auftreten, wird in einem Periodogramm angezeigt.

Dass die Periodogramm-Analyse eine adäquate Methode zur Ermittlung selbst stark gestörter Perioden ist, zeigen die Abb. Ia und b. Im zugrunde liegenden Versuch wurde das Tier durch einen 12/12 Std-Temperaturwechsel zu einer Aktivitätsperiodik induziert. Die Induktion wird im Periodogramm (Abb. 1 b) durch ein tiefes Minimum bei $\mathrm{n}=\boldsymbol{\tau}=24,0 \mathrm{Std}$ angezeigt. Im Aktogramm lässt andererseits die Folge der Aktivitätsschübe die Periodik nur undeutlich erkennen (Abb. 1a).

Bei Zeilenanzahlen, die keinem periodischen Anteil entsprechen $(n \neq \tau$ zeigt jedes Periodogramm unregelmässige Schwankungen seines "Gieräuschpegels», die um so stärker werdẹn, je größer $\mathbf{n}$ wird. Um bei der Bestimmung des Abstandes eines Minimums vom Geräuschpegel diese zufallsbedingten Schwankungen zu berücksichtigen, definierten wir D' als relativen Abstand

$$
\mathrm{D}^{\prime} \quad=\frac{\mathrm{D}}{\mathrm{S}_{\mathrm{MG}}}
$$

Dabei bedeuten D der absolute Abstand zwischen einem Minimum und dem Mittelwert des Geräuschpegels (MG) sowie sw die Standardabweichung des Geräuschpegel-Mittelwertes. MG und swi werden aus je 10 rechts und links vom Minimum liegenden Geräuschpegel-Werten gebildet. In Abb. Ib hat z.B. das Minimum bei $n=24,0$ Std einen Abstand von $\mathrm{D}^{\prime}=18$. 
Um empirische Kriterien dafür zu gewinnen, ob ein Periodogramm-Minimum zweifelsfrei eine reale Periodizität anzeigt oder auf zufallsbedingte Schwankungen des Geräuschpegels zurückgehen könnte, führten wir Simulationsexperimente durch ${ }^{11}$. Zum einen simulierten wir aperiodische Aktogramme aus Aktivitätsschüben und Ruhepausen durch Vorgabe zeitinvarianter Übergangswahrscheinlichkeiten (vgl. Einleitung). Die Länge der simulierten Aktogramme entsprach mit $\sim 2500$ Einzelwerten der Versuchsdauer, die gewählten Übergangswahrscheinlichkeiten $(p=1 / 6)$ entsprachen dem Verhalten der Höhlenkäfer. Wurden alle Werte in den Aktivitätsschüben gleich 1 gesetzt, hatten die tiefsten Periodogramm-Minima, die nach dem Bildungsgesetz der Simulation zufallsbedingt sein müssen, einen relativen Abstand von höchstens $D^{\prime} \simeq 4$. Wurde - ebenfalls in Anpassung an das Verhalten der Tiere - die Aktivitätsmenge pro Messintervall zufallsgemäß zwischen 1 und 50 variiert - betrugen die relativen Abstände der tiefsten Minima D' $\simeq 7$. Das bedeutet, daß in Periodogrammen von Messerien mit ähnlichen Parametern relative Abstände von D' $₹ 7$ mit grösserer Wahrscheinlichkeit auf zufallsbedingte Schwankungen des Geräuschpegels zurückgehen.

Um zu einer Vorstellung darüber zu gelangen, ab welchem Störungsgrad Perioden im Periodogramm nicht mehr erkennbar sind, wurden in weiteren Simulationsversuchen periodische Wertefolgen mit 17 Perioden erzeugt, wobei auf 1 Periode 143 Werte entfielen (entsprechend der Anzahl der Messwerte pro Circadianperiode). In der ungestörten Periodik wurden in der 1. Periodenhälfte alle Werte gleich 1, in der 2. Hälfte gleich 0 gesetzt. Anschließend wurde diese Wertefolge zufällig gestört, indem 0 durch 1 und umgekehrt ersetzt wurde. Die Austauschrate gibt an, wieviel Prozent der Werte der einen Phase gegen die Werte der anderen Phase ausgetauscht wurde. Bei einer Austauschrate von $50 \%$ ist die Periodik völlig ausgelöscht. Abb. 2 stellt die Beziehung zwischen Austauschrate und D' dar. Bei einem Austausch $\geqslant 45 \%$ nimmt D' Werte an, wie sie auch in Periodogrammen rein stochastisch strukturierter Wertefolgen auftreten können (s. oben), d.h. der periodische Rest ist mit einer Periodogrammanalyse nicht mehr erkennbar. Das in Abb. 1a dargestellte Aktogramm entspricht in seinem Störungsgrad einer simulierten Wertefolge mit einem Austausch von etwa 30\%.

\subsubsection{Untersuchung auf einfache stochastische Regelhaftigkeiten}

Aktogramme, in denen durch die Periodogramm-Analyse periodische Anteile nicht nachgewiesen werden können, werden auf einfache stochastische Regelhaftigkeiten untersucht. Dabei wird nur zwischen Aktivität und Ruhe unter-

1) Stochastische Verfahren zur Beurteilung von Periodogrammen liegen nicht vor (Batschelet, 1974). 
Abb. 2

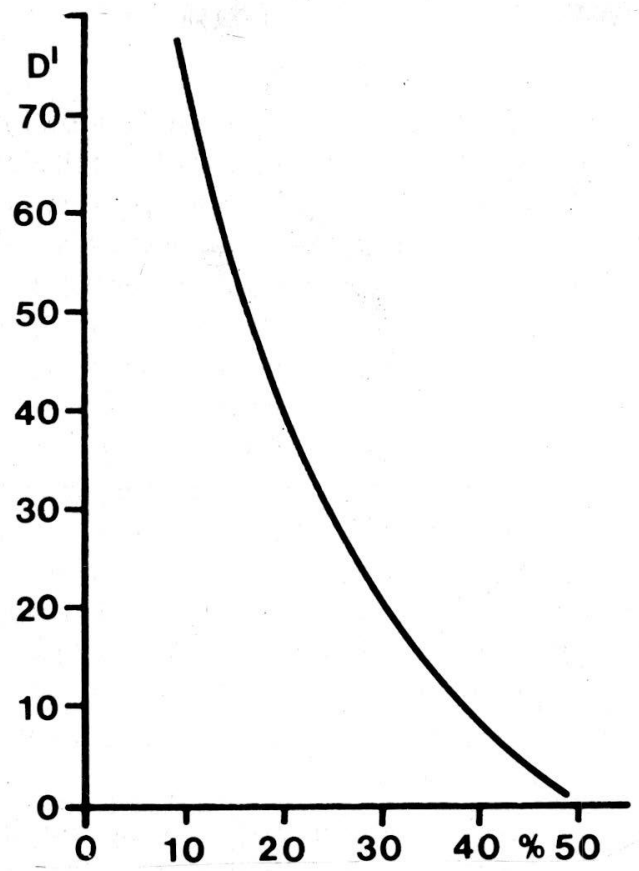

Die Abhängigkeit des relativen Geräuschpegel-Abstands des Periodogramm-Minimum (D', Ordinate) von der prozentualen Austauschrate (Abszisse) einer simulierten 111...000-Periodik. Die Austauschrate gibt an, wieviel Prozent der Werte der einen Phase gegen die Werte der anderen Phase zufällig ausgetauscht wurde. Bei einer Austauschrate von 50\% ist die Periodik ausgelöscht.

schieden, die Intensität der Aktivität bleibt unberücksichtigt (vgl. Lehmann, Neumann und Kaiser, 1974). Die Analyse umfasst:

- Die Berechnung der Koeffizienten linearer, serieller Korrelationen. Bei Fehlen periodischer Anteile ist in den Wertefolgen eine negative Korrelation zwischen aufeinanderfolgenden Schüben und Pausen mit der Regression 1 nicht enthalten. Andere korrelative Beziehungen sind aber durchaus noch denkbar; biologisch sinnvoll würden z.B. positive Korrelationen zwischen den Längen aufeinanderfolgender Schübe und Pausen erscheinen.

- Eine Untersuchung auf Konstanz des Verhaltens während eines Versuchs. Die Beurteilung erfolgt an Hand der für einzelne Versuchsabschnitte getrennt gebildeten Häufigkeitsverteilungen der Schub- sowie Pausenlängen.

- Die Berechnung der Wahrscheinlichkeiten für den Übergang von Aktivität zu Ruhe $\mathrm{p}_{\mathrm{x}_{\mathrm{R}} \rightarrow \mathrm{A}}$ bzw. von Ruhe zu Aktivität $\mathrm{p}_{\mathrm{s} \rightarrow \mathrm{K}}$ an Hand der Häufigkeitsverteilungen ( $x=$ Häufigkeitsklassen $10,2030 \ldots \mathrm{min}$ ). Dies ist nur dann zülassig, wenn die Aktogramme aperiodisch und korrelationsfrei sind, sowie Verhaltenskonstanz angenommen werden darf. Wenn diese Bedingungen gegeben sind, wird davon ausgegangen, dass sich in jedem Schub bzw. in jeder Pause eines Versuches immer wieder derselbe Determinationsprozeß vollzieht, 
der schließlich zum Ende des Schubes bzw. der Pause führt. Zur Berechnung von px wird geprüft, ob sich eine Häufigkeitsverteilung durch eine angenäherte Exponential-, Poisson- oder Normalverteilung beschreiben läßt ${ }^{1)}$. Ist dies der Fall, werden die Übergangswahrscheinlichkeiten aus der jeweiligen theoretischen Verteilung errechnet. Im andern Fall wird $\mathrm{p}_{\mathrm{x}}$ direkt aus der beobachteten Verteilung berechnet:

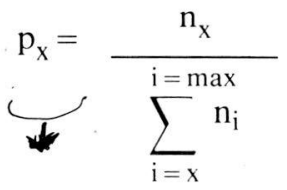

( $p_{1}=$ Übergangswahrscheinlichkeit nach einer Aktivitäts- bzw. Pausendauer

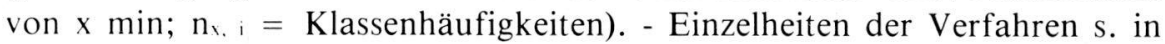
Kap. 3.1.

\section{ERGEBNISSE}

\subsection{Drei Beispiele}

Um die Verfahren im einzelnen zu erläutern, werden im folgenden drei typische Beispiele vorgestellt. Im Beispiel a wurde ein Aphaenops cerberus im 12/12 Std Licht-Dunkel-Wechsel (LD) von 0/0,5 Lux untersucht. Die Beispiele b und c sind Dauerlicht-Versuche (LL, $10^{-2}$ Lux) mit Geotrechus orpheus. In den drei Fällen sind weder in den Aktogrammen (Abb. 3a-c) noch in den Periodogrammen (Abb. 4a-c) periodische Anteile im circadianen, ultradianen $(<20 \mathrm{Std})$ oder infradianen $(>30 \mathrm{Std})$ Bereich zu erkennen. Die Minima blieben mit D' -Werten von höchstens 4 innerhalb der Grenzen der Zufallsschwankungen des Periodogramm-Geräuschpegels (s. oben).

1)a bei der Analyse auf einfache stochastische Regelhaftigkeiten nur zwischen den Zuständen der Ahtivitat und der Ruhe unterschieden wird, müssen Beginn und Ende der Aktivitätsschübe bzw. Ruhepausen definiert werden. Die Registrierung spiegelt das Verhalten nicht lückenlos wider. Kur/ere Phasen ohne Registrierungen sind nicht unbedingt echte Ruhepausen. Sie können auch dadurch bedingt sein, dass das Tier zwar laufaktiv ist, den Messstrahl aber zufällig nicht kreuzt. Dies war offensichtlich bäufiger der Fall, da in vielen Aktogrammen kurze Abschnitte ohne Regiuricrung gchäuft innerhalb von Phasen starker Aktivität auftreten (z. B. Pfeile in Abb. 3a). In der Hatufigkeimerteilung der Abb. 7b geht das Maximum in der 1. Klasse möglicherweise auf solche "uncihten" Ruhepausen surück: die Verteilung der realen Ruhepausen folgt einer Normalverteilung mit cincm Häufigkeitsbereich in mittleren Klassen (Abb. 7i)

1) I chmann. Neumann 4. Kaiser (1974) überprüften die Häufigkeitsverteilungen der Winkerkrabben-Aktogramme nur auf Übereinstimmung mit der Exponentialfunktion $y=a \cdot{ }^{\prime}$. Da in der Neurophysiologie aucb andere Verteilungen bekannt sind (z. B. bei Inter-

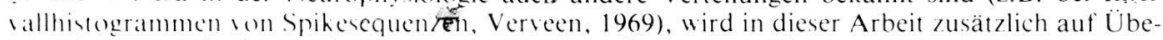
reimstimmung mit cince Poisson-oder Normalverteilung untersucht 


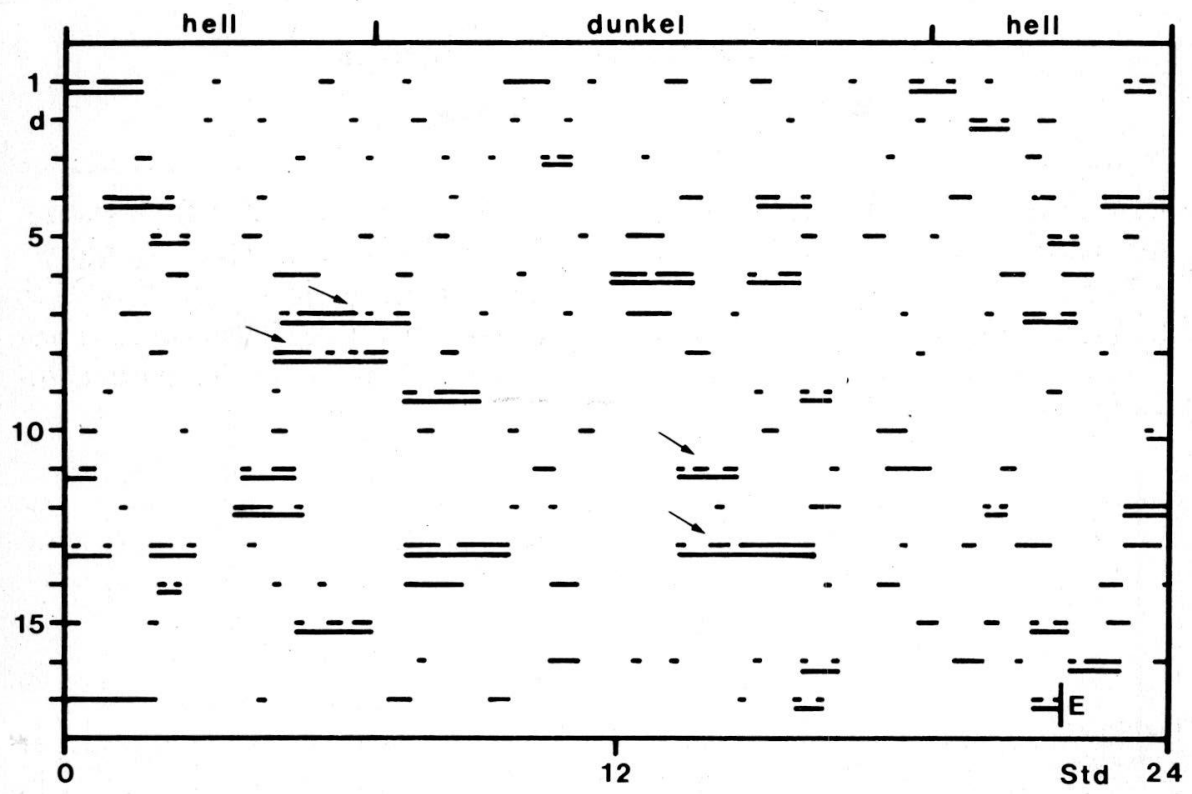

Abb. 3a - Aktogramme eines Aphaenops cerberus im LD von 0/0,5 Lux und in $9^{\circ} \mathrm{C}$ Konstanttemperatur. $\mathrm{d}=$ Versuchstage. Linien: Aktivitätsregistrierungen, die durch kurze Abschnitte ohne Registrierung unterbrochen sind, werden zu einem Aktivitätsschub zusammengefasst (jeweils die untere Linie). Pfeile: Beispiele für gehäuftes Auftreten kurzer Abschnitte ohne Registrierung in Phasen stärkerer Aktivität.

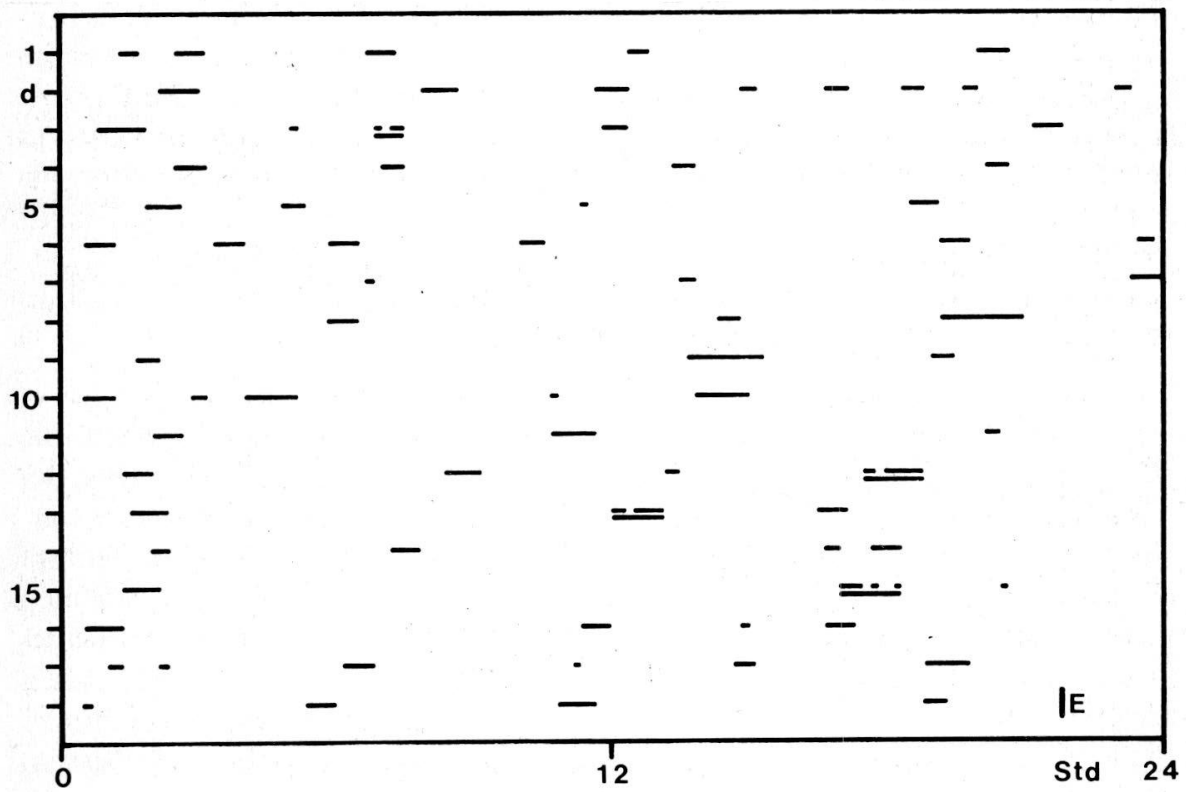

Abb. 3b. Aktogramm eines Geotrechus orpheus im I. I. von $10^{2} 1$ ux und 9 ( Komstantlemperat. tur. 


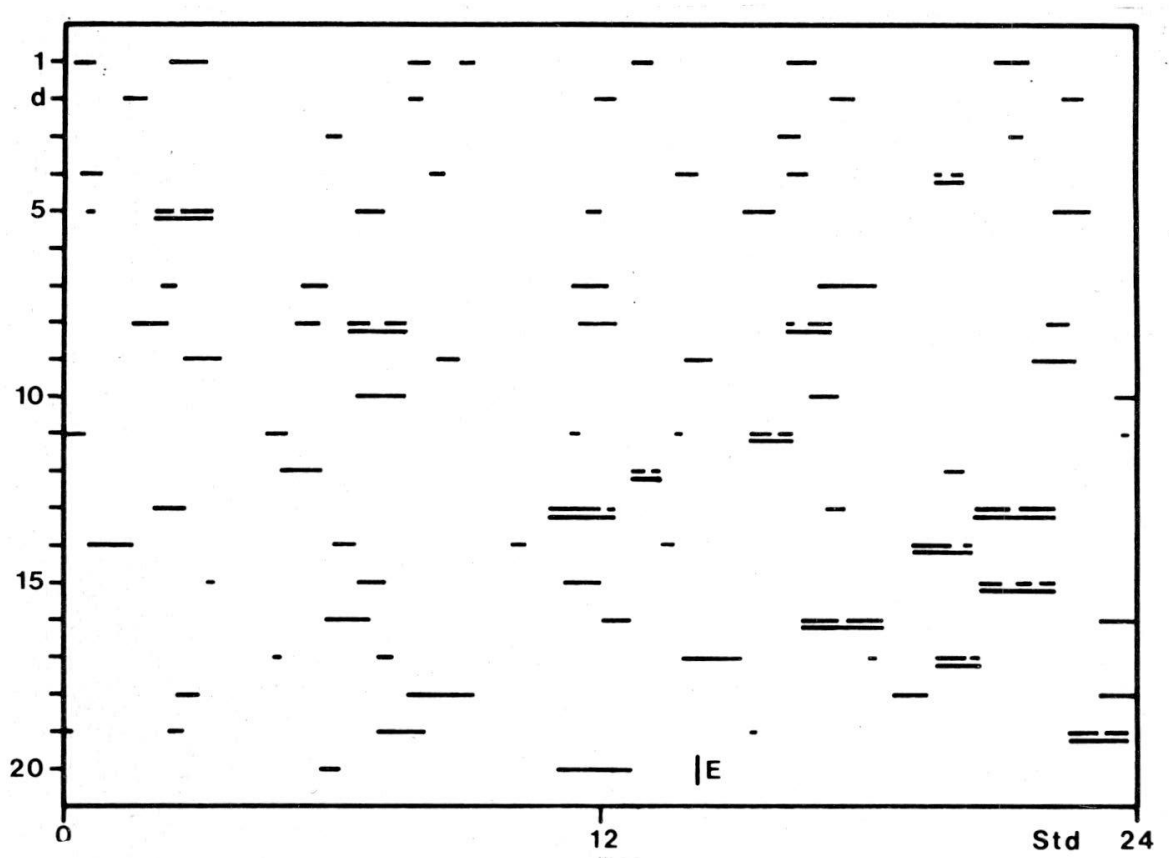

Abb. 3c - Aktogramm eines Geotrechus orpheus im LL von $10^{-2}$ Lux und $9^{\circ} \mathrm{C}$ Konstanttemperatur.

Nach Beobachtungen des Verhaltens der Tiere im Aktographen können wir davon ausgehen, dass sich ein Tier praktisch immer dann in Ruhe befindet, wenn mindestens vier 10 min-Intervalle ohne Registrierung aufeinanderfolgen. Ein Aktivitätsschub gilt deshalb nur dann als beendet, wenn diese Bedingung erfüllt ist. Reale Ruhepausen kleiner als $4 \times 10$ min können also aus messtechnischen Giünden nicht erkannt werden.

Die I ängen der Abchnitte mit lückenloser Registrierung werden mit A', die Längen der Abschnitte ohne Registrierung mit R', die Längen der nach dem Kriterium bestimmten Aktivitätsschübe und Ruhepausen mit A bzw. R abgekürzt. Aus den Aktogrammen der drei Beispiele (Abb. 3a-c) können sowohl $A^{\prime}$ und $R^{\prime}$ als auch $A$ und $R$ bestimmt werden.

Tab. 1 enthält die Koeffizienten der untersuchten seriellen Korrelationen. Sie sind in einigen Fällen auf dem 5\%- oder 1\%- Niveau signifikant. Das Bestimmtheitsmass $B=100 \cdot r^{2}$, das angibt, wieviel Prozent der Gesamtstreuung der einen Variablen durch die Gesamtstreuung der anderen bedingt ist (Sachs, 1974), bleibt aber stets so klein $(\leqslant 13 \%)$, dass die Korrelationen nicht relevant für die Entstehung der Folgen aus Aktivitätsschüben und Ruhepausen sein können. Es darf als weitgehende Annäherung an die Wirklichkeit angenommen werden, dass die Ereignisse, die zum Übergang von Aktivität zu Ruhe bzw. von Ruhe zu Aktivität führen, sich gegenseitig nicht beeinflussen. Die Folgen von Aktivität und Ruhe sind stochastisch strukturiert.

Im nächsten Schritt wird auf Verhaltenskonstanz geprüft. In den Beispielen a 

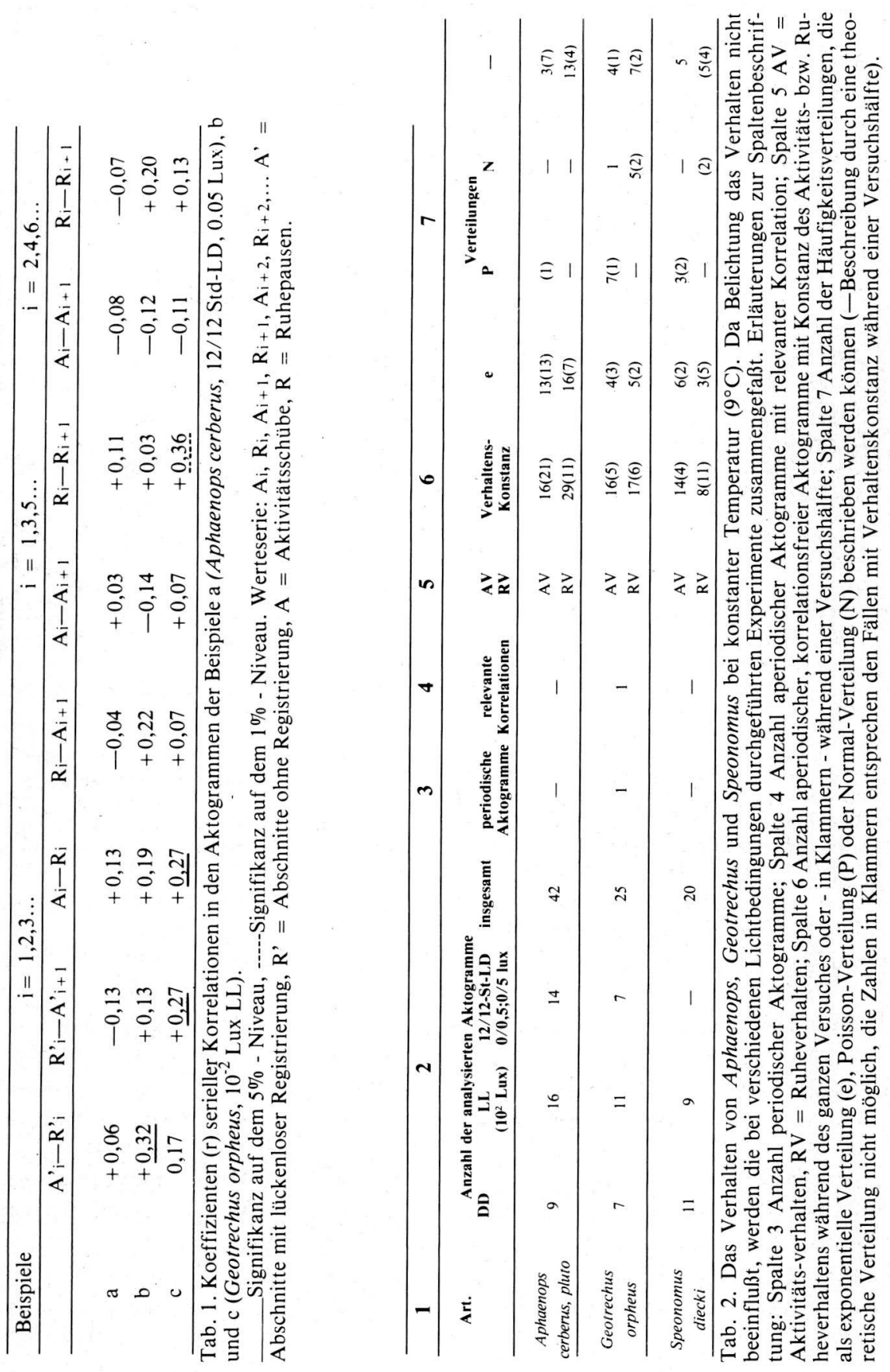

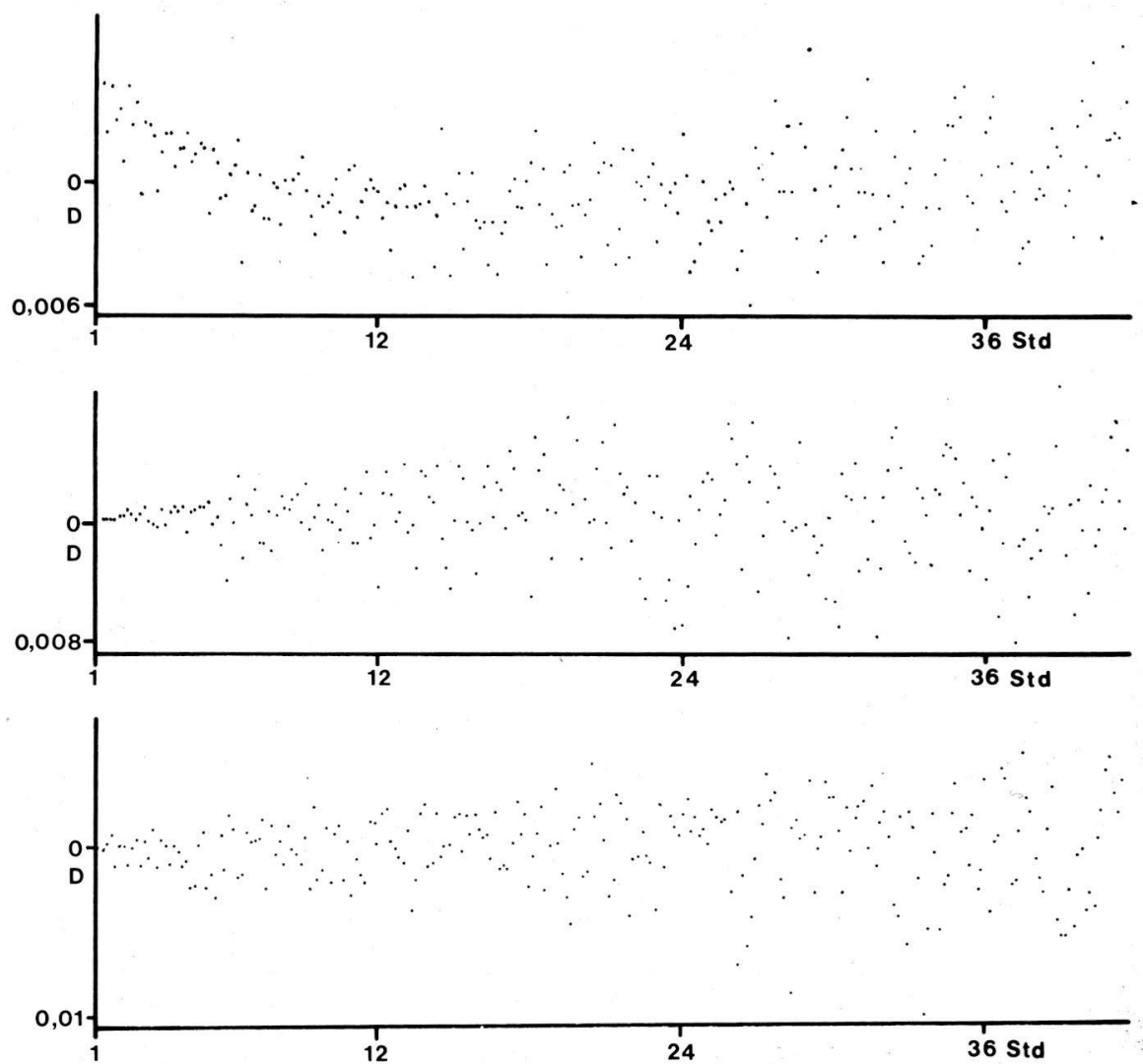

Abb. 4a, b, c - Periodogramme der in Abb. 3a, b, c, dargestellten Aktogramme aus den Originalwerten (unter Berücksichtigung der Aktivitätsintensität) errechnet. D (Ordinate) ist der Abstand der Periodogrammwerte vom Mittel aller Periodogrammwerte. In keinem der Periodogramme wird eine Periodik angezeigt.

und $\mathrm{b}$ sind die für die 1. und 2. Versuchshälfte getrennt gebildeten Häufigkeitsverteilungen jeweils einander so ähnlich (Abb. 5c, d, 6c-e), dass Konstanz des Aktivitäts- und Ruheverhaltens angenommen werden kann. Im Beispiel c weist die A-Verteilung in der 1. Versuchshälfte ein deutliches Maximum in der 3. Längenklasse, in der 2. Versuchshälfte in der 1. Längenklasse auf. Eine weitere Unterteilung in vier gleiche Versuchsabschnitte verdeutlicht, dass sich das Tier in der 2. Versuchshälfte konstant verhält, während sich die A-Verteilungen auch im 1. und 2. Versuchsviertel relativ stark voneinander unterscheiden (Abb. 7c-f). Im Beispiel $\mathrm{c}$ ist also das Aktivitätsverhalten im Gegensatz zum Ruheverhalten nur in der 2. Versuchshälfte konstant.

Die A- und R-Verteilung des Beispiels a sowie die A-Verteilung der 2. Versuchshälfte des Beispiels c lassen sich exponentiellen Verteilungen des Typs 

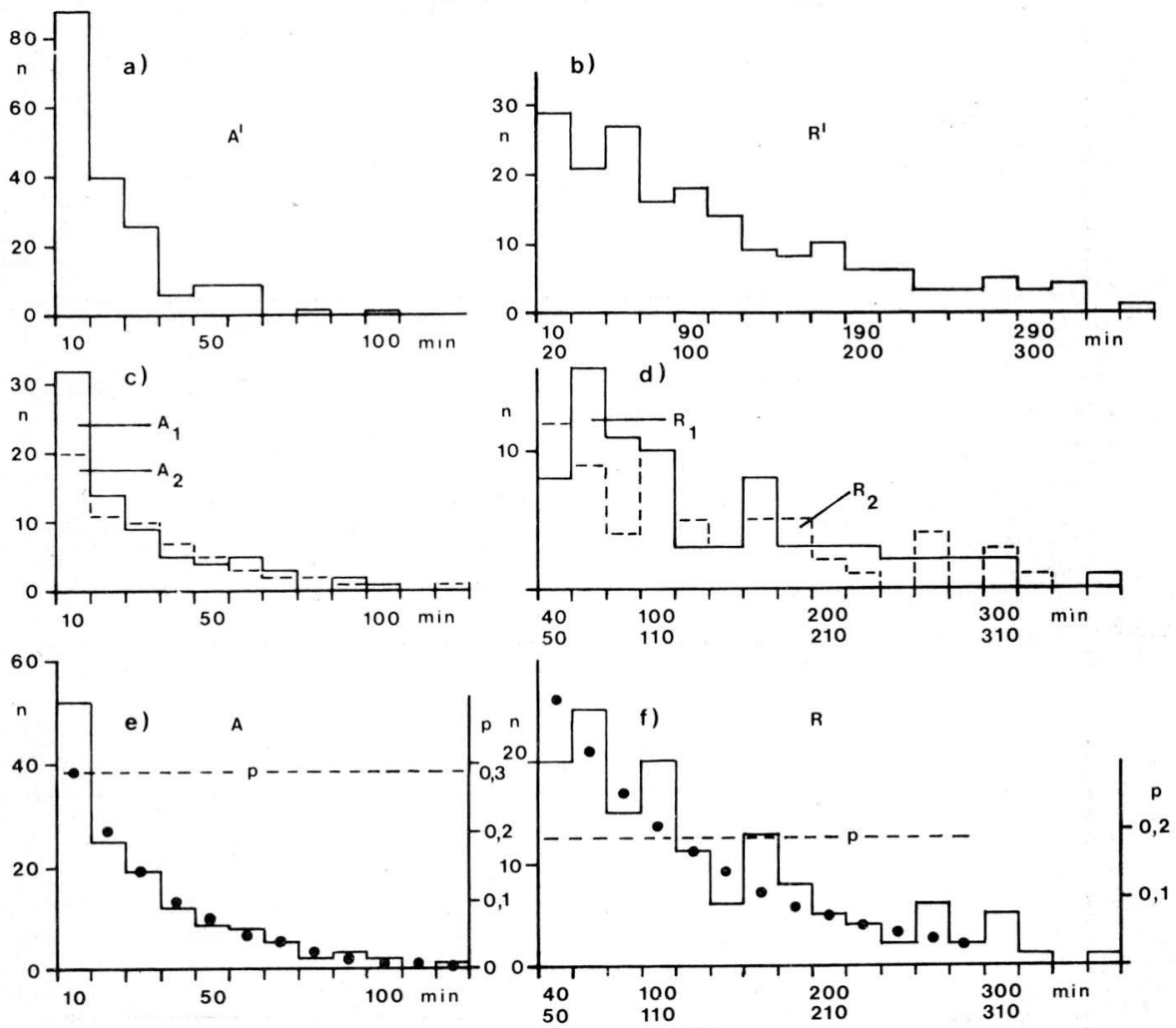

Abb. 5 - Häufigkeitsverteillungen des Beispiels a (Aphaenops cerberus, LD, 0/0,5 Lux, vgl. Abb. 3a, 4a). a: Längen der Abschnitte mit lückenloser Registrierung (A').

b: I ängen der Abschnitte ohne Registrierung (R').

¿: I ängen der Aktivitätsschübe in der 1. $\left(A_{1}\right)$ und 2. Versuchshälfte (A2). d: Längen der Ruhepausen in der 1. $\left(R_{1}\right)$ und 2. Versuchshälfte $\left(R_{2}\right)$. e: Längen der Aktivitätsschübe des ganzen Versuchs (A). - f: Längen der Ruhepausen des ganzen Versuchs (R). - In den Vèrteilungen b, $d, f$ sind je 2 I ängenklassen zusammengefasst.

Punkteverlauf in e und $\mathrm{f}$ : angenäherte exponentielle Verteilung ( $\chi^{2}=0,60$ bzw. 0,09). Rechte ()rdinate in e und f: Übergangswahrscheinlichkeit p für die jeweilige Klassenbreite.

$y=a \cdot e^{-1 \cdot}$. annähern $\left(\chi^{2}\right.$-Test, Irrtumswahrscheinlichkeit bei Ablehnung der Null-Hypothese $\alpha \leqq 0.05$ ). Das bedeutet, dass die jeweilige Übergangswahrscheinlichkeit $\mathrm{p}, \overline{\mathrm{zu}}$ jedem Zeitpunkt innerhalb der Schübe (bzw. Pau sen) gleich gross ist (Zeitinvarianz von $\mathrm{p}_{\mathrm{v}}$, Abb. 5e, f, 7h, vgl. Lehmann, Neumann, Kaiser, 1974). Im Beispiel b lässt sich die A-Verteilung durch eine Poisson-Funktion, die R-Verteilung durch eine Normalverteilung beschreiben (Abb. 6f, g). Auch im Beispiel c folgt die R-Verteilung einer Normalverteilung (Abb. 7 i). In diessen Fällen wird die Übergangswahrscheilichkeit $p_{x}$ mit der Dauer der Schübe bzw. Pausen grösser (vgl. Abb. 6 f, g, 7i). Aus dem Fehlen horrelativer Beziehungen muss aber gefolgert werden, dass $\mathrm{p}$ 、 wie bei den exponentiellen Verteilungen nicht von der Länge der vorausgehenden Schübe 

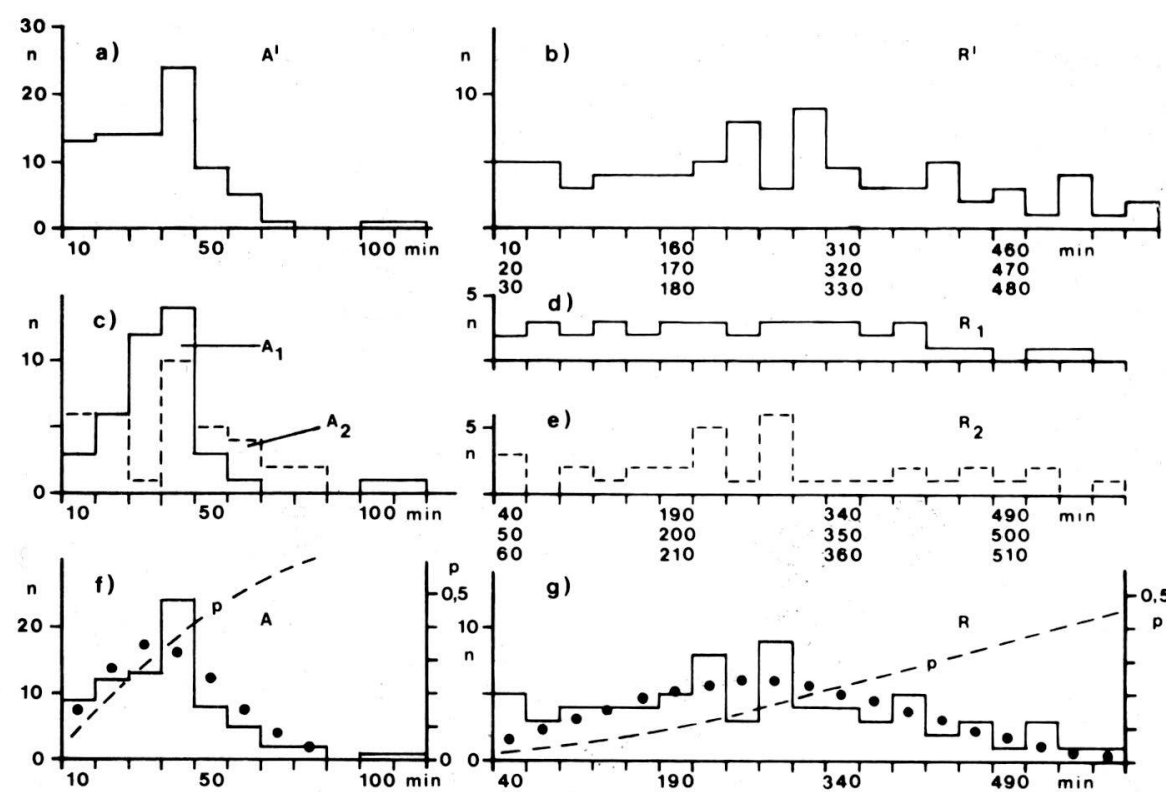

g)

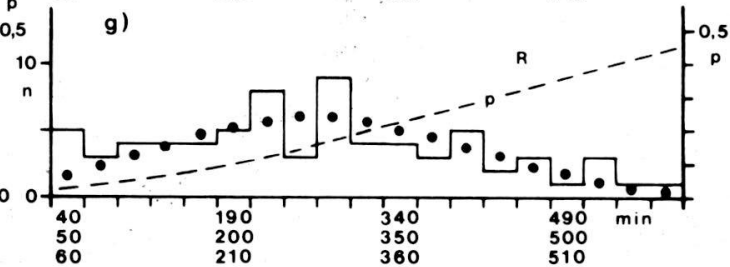

Abb. 6 - Häufigkeitsverteilungen des Beispiels b (Geotrechus orpheus, LL, 10 ${ }^{-2}$ Lux). In b. d, e und $\mathrm{g}$ werden je 3 Längenklassen zusammengefasst. Punkteverlauf in $\mathrm{f}$ : angenäherte PoissonVerteilung

$\left.\alpha \chi^{2}=0,15\right)$, in g: angenäherte Normal-Verteilung $\left(\alpha_{\chi} \chi^{2}=0,20\right)$. - Im übrigen entsprechend der
Legende von Abb. 5 .

und Pausen bestimmt wird. - Auch im Beispiel c dürfte in der 1. Versuchshälfte $\mathrm{p}_{\mathrm{x}_{\mathrm{A} \rightarrow \mathrm{R}}}$ die Tendenz haben, mit der Dauer eines Schubes grösser zu werden (das Maximum der Verteilung liegt in der 3. bzw. 5. Klasse, vgl. Abb. 7 c, d).

Bei grossen mittleren Schub- und Pausenlängen werden - um für den statistischen Test ausreichend grosse Klassenhäufigkeiten zu haben - benachbarte Klassen zusammengefasst, bei mittleren Längen von $100<\bar{A}(\bar{R})<150$ min je 2, bei $\bar{A}(\bar{R})>150$ min je 3 benachbarte Klassen. Die in den Abb. 5, 6, 7 angegebenen Übergangswahrscheinlichkeiten gelten für die jeweils gewählte Klassenbreite. - Darüber hinaus mussten für den Anpassungstest bei schwach besetzten Klassen weitere Zusammenfassungen erfolgen, die in den Abb. nicht angegeben sind. - Die angenäherten Funktionen beșchreiben in den Beispielen mindestens $95 \%$ der jeweiligen Gesamthäufigkeit. Der Rest entfällt auf höhere Klassen, die in manchen Fällen zu häufig auftreten (vgl. z.B. Abb. 5 f). Dies muss nicht unbedingt bedeuten, dass $p$, die Tendenz hat, bei grosser Aktivitäts- bzw. Ruhedauer kleiner zu werden. Die Abweichungen können auftreten, weil sehr kurze Aktivitätsschübe nicht immer zu Registrierungen führen; auch zu viele lange Aktivitätsschübe sind zu erwarten, weil unter den 

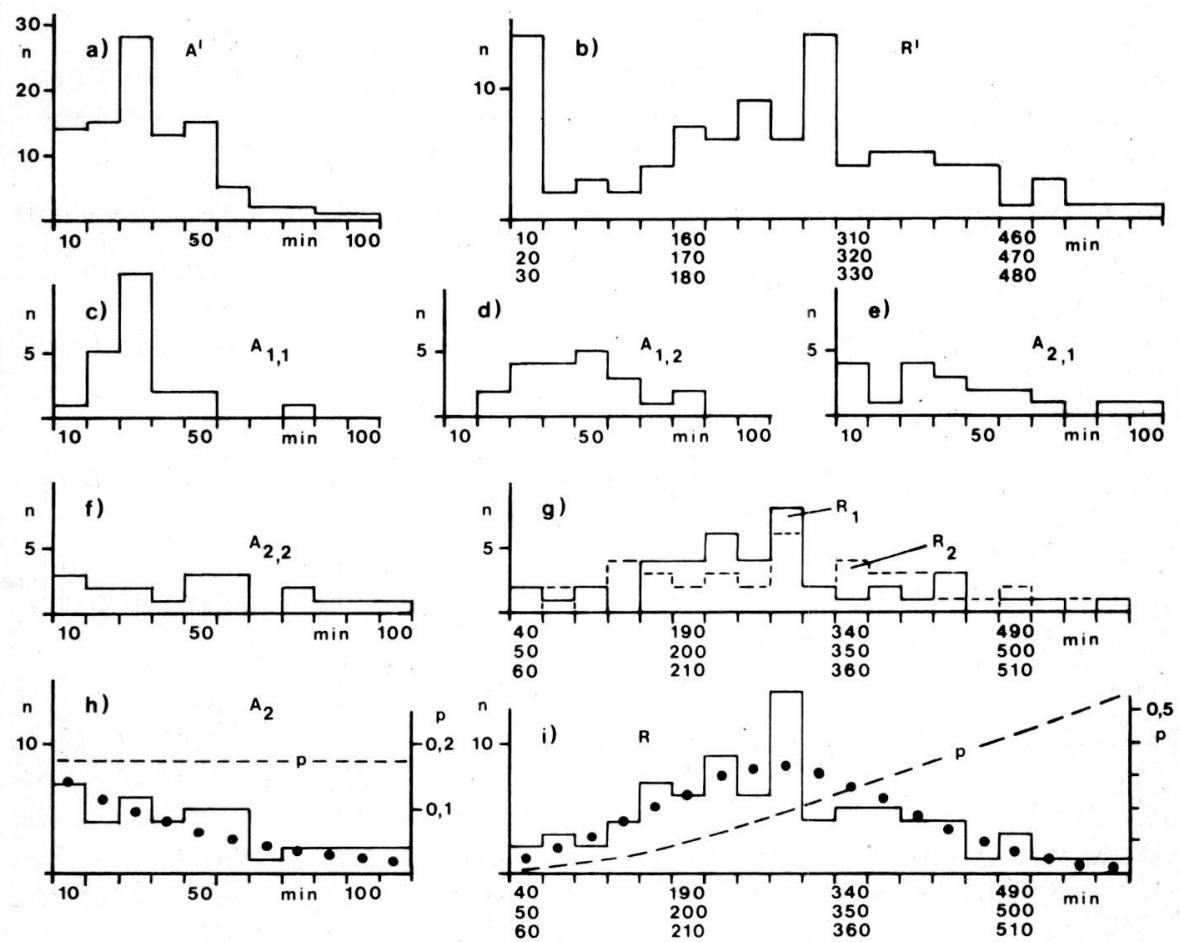

Abb. 7 - Häufigkeitsverteilungen des Beispiels c (Geotrechus orpheus, LL, $10^{-2}$ Lux). c, d, e, f: Häufigkeitsverteilungen der Schublängen des 1., 2., 3. und 4. Versuchsviertels $\left(\mathrm{A}_{1,1} ; \mathrm{A}_{1,2} ; \mathrm{A}_{2,1}\right.$; $\mathrm{A}_{2,2}$ ). In $\mathrm{b}, \mathrm{g}$ und i wurden je 3 Längenklasser zusammengefasst. Punkteverlauf in h: angenäherte Exponentialverteilung ( $\left.\alpha \chi^{2}=0,45\right)$, in i:angenäherte Normal-Verteilung $\left(\alpha \chi^{2}=0,40\right)$. - Im übrigen entsprechend der Legende von Abb. 5.

kurzen Abschnitten ohne Registrierung, die nach der oben gegebenen Definition generell zu Aktivitätsschüben geschlagen werden, sich echte kurze Ruhepausen befinden können.

\subsection{Periodogrammanalysen}

Die Aktogramme der bei konstanter Temperatur $\left(9^{\circ} \mathrm{C}\right)$ im Dauerdunkel (DD), Dauerlicht (LL, 10-2 Lux), Licht-Dunkel-Wechsel (LD) untersuchten Tiere lassen - bis auf eine Ausnahme - keine periodischen Anteile erkennen (Tab. 2). Sie bestehen aus unterschiedlich langen Aktivitätsschüben und Ruhepausen, die scheinbar regellos aufeinanderfolgen.

Die Periodogramme weisen im untersuchten Bereich von 50 min bis 42 Std nur sehr flache Minima auf, deren D'-Werte stets kleiner als 6 sind. Ihre Zugehörigkeit zum Geräuschpegel kann also nicht ausgeschlossen werden. Würden 
durch sie tatsächlich periodische Wertefolgen angezeigt, so entsprächen diese in ihrem Störungsgrad 111...000-Perioden mit einer Austauschrate von mindestens $42 \%$ (vgl. Abb. 2).

Nur ein Geotrechus-Versuch (DD) macht eine Ausnahme. Im Periodogramm liegt bei $\mathrm{n}=23,8$ Std ein tieferes Minimum ( $D^{\prime}=9$ ). Auch das Aktogramm selbst lässt eine Periodik erkennen: die Aktivitätsschübe treten in etwa 24 Std voneinander entfernten Perioden gehäuft auf. Die Periodizität geht möglicherweise auf eine Induktion durch einen äusseren Umweltzyklus zurück.

\subsection{Korrelationsanalysen}

Mit Ausnahme des schwach periodischen Geotrechus-Aktogrammes wurden alle Aktogramme auf die seriellen Korrelationen $A_{i}-R_{i}$ und $R_{i}-A_{i+1}$ untersucht. Stichprobenartig wurden von einigen Aktogrammen auch die Korrelationen zwischen aufeinanderfolgenden Schüben bzw. Pausen errechnet (Erläuterungen in Tab. 1).

In etwa 12\% der Fälle sind die Korrelationen auf dem 1\%-Niveau signifikant. Bis auf eine Ausnahme ist das Bestimmtheitsmass B aber so klein $(1 \times 14 \%$, $1 \times 13 \%$, sonst unter $10 \%$ ), dass diese Korrelationen für die Entstehung der Sequenzen aus Aktivitätsschüben und Ruhepausen nicht relevant sein können. Die Ausnahme betrifft ein weiteres Geotrechus-Aktogramm (DD) mit $\mathrm{r}_{\mathrm{R} i}-\mathrm{A}_{\mathrm{i}+1}$ $=+0,64$ und $\alpha<0,1 \%$. Nur in diesem Fall wird die Korrelation auch erkennbar, wenn die Wertepaare in ein Koordinatensystem eingetragen werden.

Wir schliessen aus den Periodogramm- und Korrelations-Analysen, dass - bis auf die beiden erwähnten Ausnahmen - in den Aktogrammen weder ultradiane, circadiane noch infradiane periodische Komponenten enthalten sind und dass darüber hinaus die Übergänge von Aktivität zu Ruhe bzw. von Ruhe zu Aktivität von der Dauer der vorausgehenden Schübe und Pausen unabhängig sind.

\subsection{Untersuchung auf Verhaltenskonstanz}

Von den insgesamt 86 analysierten Aktogrammen war in 46 Fällen das Aktivitätsverhalten während des ganzen Versuchs konstant, in weiteren 30 Fällen wenigstens während einer Hälfte. Entsprechendes gilt für 54 bzw. 27 Aktogramme in bezug auf das Ruheverhalten (Tab. 2).

\subsection{Häufigkeitsverteilungen und Übergangswahrscheinlichkeiten}

Ein Einfluss der verschiedenen Lichtbedingungen (DD, LL, LD) auf die Höhe bzw. die Zeitabhängigkeit der Übergangswahrscheinlichkeit ist bei Aphaenops 
und Geotrechus nicht erkennbar. Die Tiere verhalten sich dem Licht gegenüber völlig indifferent (Speonomus wurde nur in DD untersucht). Es können deshalb die bei den verschiedenen Lichtbedingungen durchgefürten Untersuchungen zusammen-gefasst werden (Tab. 2).

$\mathbf{7 4 \%}$ der A- und $57 \%$ der R-Verteilungen können von einer exponentiellen Verteilung, einer Poisson- oder Normalverteilung nicht unterschieden werden (Tab. 2). Bei Aphaenops treten fast ausschliesslich exponentielle Verteilungen (zeitinvariante Übergangswahrscheinlichkeiten) auf. Bei Geotrechus und Speonomus kommen neben exponentiellen Verteilungen häufiger Poissonund Normalverteilungen vor (mit der Dauer der Schübe bzw. Pausen zunehmende Übergangswahrscheinlichkeiten). - Der Bereich der Übereinstimmung mit der angenäherten Funktion umfasst mindestens $85 \%$ der jeweiligen Gesamthäufigkeit, in den meisten Fällen ist er grösser als $90 \%$.

Von den Verteilungen, die sich nicht durch eine der drei theoretischen Verteilungen beschreiben lassen, wird $p_{x}$ direkt aus den beobachteten Klassenhäufigkeiten errechnet und für die Arten klassenweise gemittelt. Wegen starker zufallsbedingter Schwankungen in den höheren, relativ schwach besetzten Klassen ist in Abb. $8 \bar{p}_{x}$ nur für die jeweils ersten 7 Klassen dargestellt. Die Unterschiede zu den aus den theoretischen Funktionen errechneten Wahrscheinlichkeiten sind z.T. beträchtlich. So zeigt $\overline{\mathrm{p}}_{\mathrm{x}_{\mathrm{A} \rightarrow \mathrm{R}}}$ bei Aphaenops in den ersten beiden Klassen und bei Geotrechus in mittleren Klassen ein deutliches Maximum. $\overline{\mathrm{p}}_{\mathrm{R}_{\mathrm{R}} \rightarrow \mathrm{A}}$ weist bei Geotrechus ein Minimum im Bereich mittlerer Klassen auf. Am ehesten entsprechen $\overline{\mathrm{p}}_{\mathrm{x}_{\mathrm{R} \rightarrow \mathrm{A}}}$ bei Aphaenops (annähernd konstant), und $\overline{\mathrm{p}}_{\mathrm{x}}{ }_{\mathrm{A} \rightarrow \mathrm{R}}$ sowie $\overline{\mathrm{p}}_{\mathrm{x}_{\mathrm{R}} \rightarrow \mathrm{A}}$ bei Speonomus (annähernd stetige Zunahme mit der Zeit) den sich auch aus den berücksichtigten theoretischen Funktionen ergebenden Übergangswahrscheinlichkeiten.

\subsection{Die Durchschnittswerte der mittleren Schub- und Pausenlängen (Tab. 3).}

Geotrechus orpheus unterscheidet sich bei der Untersuchungstemperatur von $9^{\circ} \mathrm{C}$ in bezug auf die Durchschnittswerte der mittleren Schub- und Pausenlängen $(\overline{\mathrm{A}}, \overline{\mathrm{R}}$,$) deutlich von Aphaenops und Speonomus. Seine Schübe sind im$ Mittel kürzer, seine Pausen erheblich länger. Aphaenops und Speonomus unterscheiden sich andererseits nicht voneinander.

Aus praktischen (iründen werden in den folgenden Kapiteln anstelle der Übergangswahrscheinlichkeiten die Mittelwerte der Schub- und Pausenlängen untersucht. Durch den Mittelwert ist sowohl cinc exponentielle als auch eine Poisson-Verteilung eindeutig charakterisiert, in Verbindung mit der Standardabweichung auch eine Normalverteilung. 

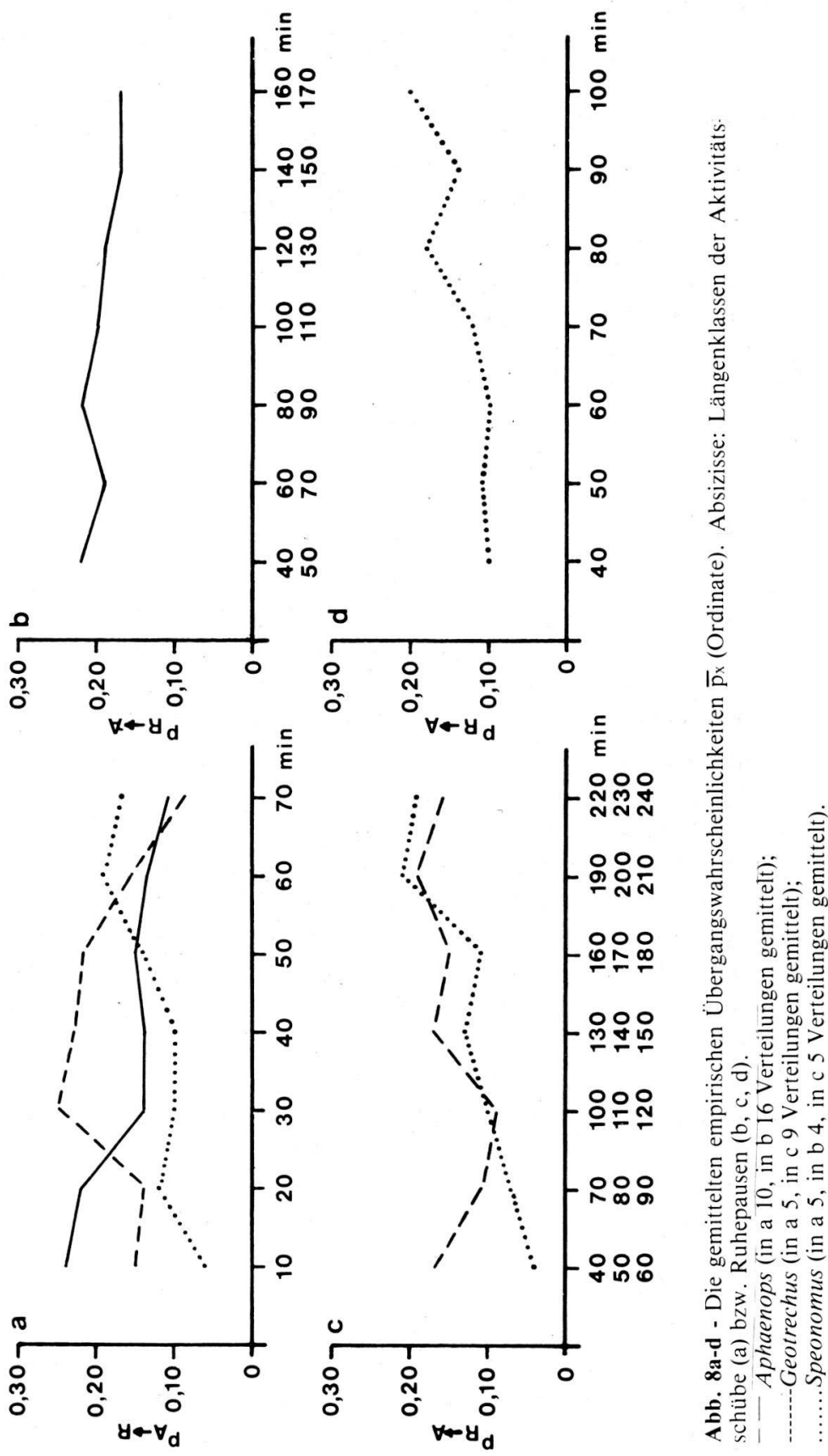


\begin{tabular}{lcc}
\hline & Schublänge (min) & Pausenlänge (min) \\
\hline Aphaenops-Arten & $78 \pm 33$ & $153 \pm 77$ \\
Geotrechus orpheus & $57 \pm 23$ & $263 \pm 65$ \\
Speonomus diecki & $84 \pm 47$ & $164 \pm 56$ \\
\hline
\end{tabular}

Tab. 3. Die Durchschnittswerte der mittleren Schub- bzw. Pausenlängen mit Standardabweichungen. Anzahl der Tiere s. Tab. 2.

\subsection{Nichtkonstanz des Verhaltens und Korrelation zwischen mittleren Schub- und Pausenlängen.}

Nichtkonstanz des Aktivitäts- und/oder Ruheverhaltens ist möglicherweise die Folge endogen bedingter Veränderungen der Übergangswahrscheinlichkeiten. Verändert sich $p_{x}$ im Ablauf der Versuche regelhaft? Diese Frage soll an Hand der mittleren Schub- und Pausenlängen $(\overline{\mathrm{A}}, \overline{\mathrm{R}})$ geprüft werden, indem diese Mittelwerte getrennt für die 1. und 2. Versuchshälfte gebildet werden $\left(\overline{\mathrm{A}}_{1}, \overline{\mathrm{A}}_{2}, \overline{\mathrm{R}}_{1}, \overline{\mathrm{R}}_{2}\right)$. $\overline{\mathrm{A}}$ zeigt bei keiner Art eine deutliche Tendenz, sich im Ablauf der Versuche regelhaft zu verändern (Abb. 9a-c). Entsprechendes gilt bei Aphaenops und Geotrechus auch für $\overline{\mathrm{R}}$. Bei Speonomus aber ist $\overline{\mathrm{R}}$ in 18 von 20 Fällen in der 2. Versuchshälfte grösser $\left[\bar{R}_{1} /\left(\bar{R}_{1}+\bar{R}_{2}\right)<0,5\right.$, vgl. Abb. $\left.9 \mathrm{c}\right]$.

Bei Aphaenops fällt auf, dass eine Verkürzung von $\overline{\mathrm{A}}$ häufiger mit einer Verlängerung von $\bar{R}$ einhergeht und umgekehrt: die Quotienten $\bar{A}_{1} /\left(\bar{A}_{1}+\bar{A}_{2}\right)$ und $\overline{\mathrm{R}}_{1} /\left(\overline{\mathrm{R}}_{1}+\overline{\mathrm{R}}_{2}\right)$ sind schwach negativ miteinander korreliert $(r=-0,62$; $\mathrm{n}=42 ; \alpha<0,1 \%$, vgl. Abb. 9a). Dies bedeutet für den Fall zeitinvarianter Übergangswahrscheinlichkeiten, die bei Aphaenops vorherrschen, dass sich $\mathrm{p}_{\mathrm{A}} \mathrm{R}$ und $\mathrm{p}_{\mathrm{R}} \mathrm{A}_{\mathrm{A}}$ häufiger gegensinnig spontan verändern.

\subsection{Der Einfluss von Umweltfaktoren}

12/12 Std-LD beeinflussen die Aktivität von Aphaenops und Geotrechus nicht (Aphaenops: 0/0,5 Lux, 10 Tiere; 0/5 Lux: 8 Tiere; Geotrechus: 0/0,5 Lux, 9 Tiere; Speonomus wurde nicht untersucht). Die Möglichkeit eines Proportionaleffektes wurde an je 12 Aphaenops- Tieren in folgenden Versuchsprogrammen getestet:

7 Tage DD $\rightarrow 7$ Tage 0,5 Lux LL $\rightarrow 7$ Tage DD sowic

7 Tage DD $\rightarrow 7$ Tage 5 Lux LL $\rightarrow 7$ Tage DD.

Eine regelhafte Veränderung von $\bar{A}$ oder $\bar{R}$ durch das l icht war in keinem Fall erkennbar.

Im Gegensatz dazu induzieren 12/12 Std-Temperaturwechsel (TW) bei den untersuchten Arten der 3 Gattungen eine deutliche Aktivitätsperiodik (Abb. 


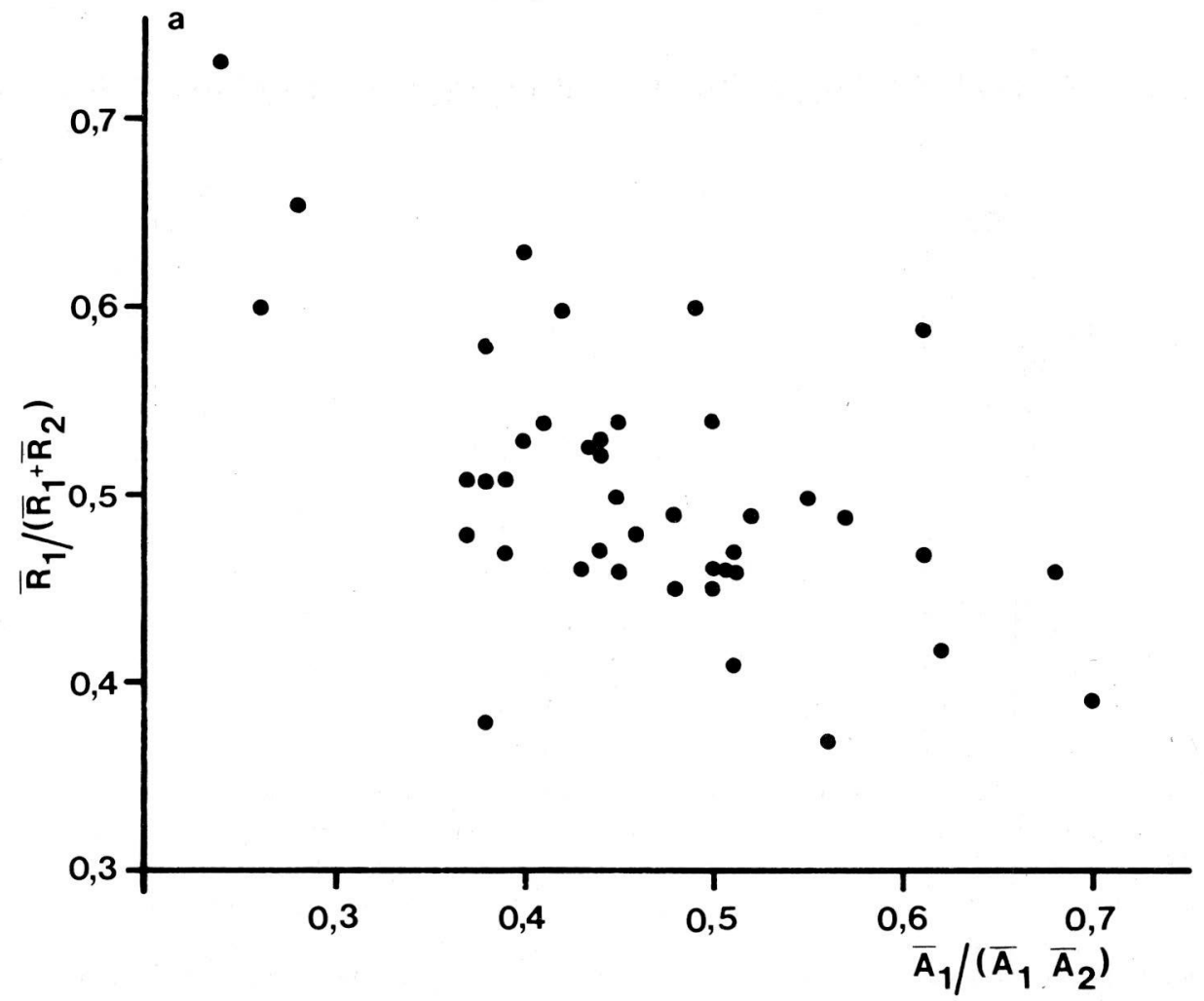

Abb. 9a - Aphaenops cerberus und $A$. pluto. Keine regelhaften Veränderungen von $\overline{\mathrm{A}}$ und $\overline{\mathrm{R}}$ im Verutuchablauf. Schwache negative Korrelation zwischen der Veränderung von $\bar{A}$ und $\bar{R}$ ( ) $-0,62, \alpha<0,1 \% 0$.

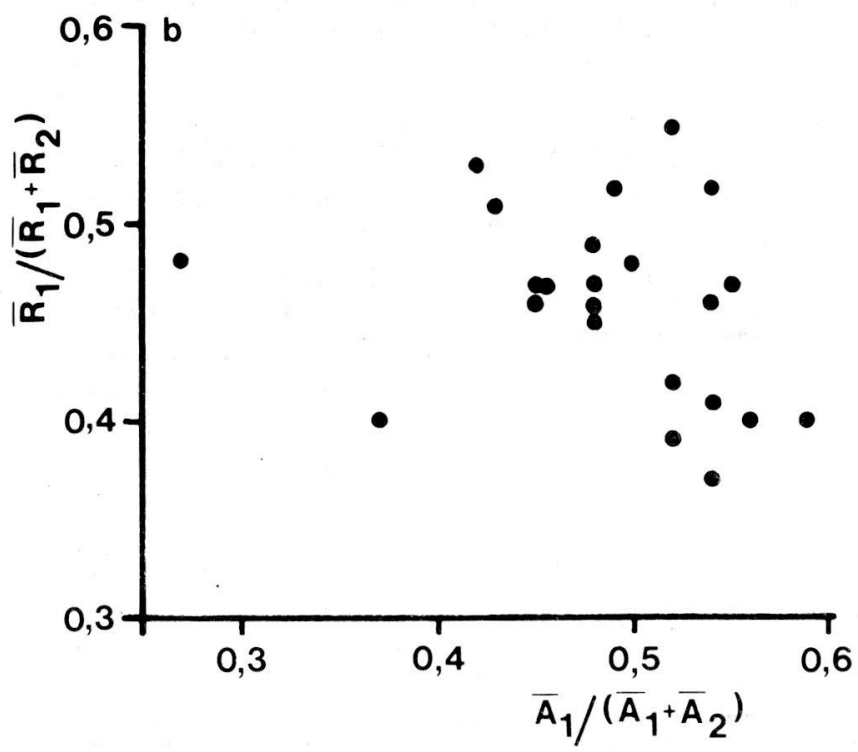

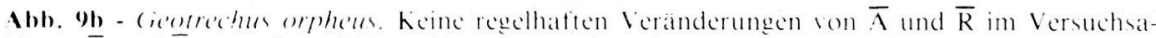
Wlate. $\bar{T}$ und $\bar{R}$ lerandern ich unabhangig boncinander. 


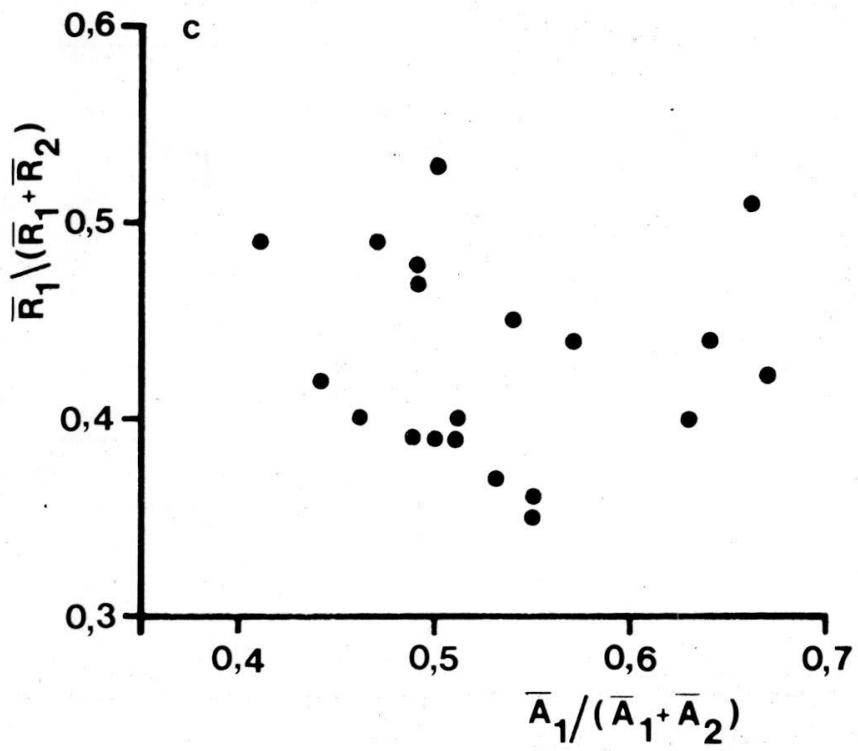

Abb. 9c - Speonomus diecki. $\overline{\mathrm{R}}$ wird in 18 von 20 Fällen in der 2 . Versuchshälfte länger. $\overline{\mathrm{A}}$ verändert sich nicht regelhaft.

Abb. 9a, b, c - Die Veränderungen der mittleren Schublänge $(\overline{\mathrm{A}})$ und der mittleren Pausenlänge $(\bar{R})$ im Ablauf der Versuche bei konstanter Temperatur (s. Tab. 2), dargestellt an Hand der Quotienten $\overline{\mathrm{A}}_{1} /\left(\overline{\mathrm{A}}_{1}+\overline{\mathrm{A}}_{2}\right)$ [Abszisse] und $\overline{\mathrm{R}}_{1} /\left(\overline{\mathrm{R}}_{2}+\overline{\mathrm{R}}_{2}\right)$ [Ordinate]. $\overline{\mathrm{A}}_{1}$ ist die mittlere Schublänge für die 1 . Hälfte, $\bar{A}_{2}$ für die 2 . Hälfte des Versuchs. Entsprechendes gilt für $\bar{R}_{1}$ und $\bar{R}_{2}$.

\begin{tabular}{|c|c|c|c|c|}
\hline & \multicolumn{2}{|c|}{$12 / 12 \mathrm{Std}$} & \multicolumn{2}{|c|}{$24 / 24 \mathrm{Std}$} \\
\hline & $\begin{array}{l}\text { Aphaenops- } \\
\text { Arten }\end{array}$ & $\begin{array}{l}\text { Geotrechus } \\
\text { orpheus }\end{array}$ & $\begin{array}{l}\text { Speonomus } \\
\text { diecki }\end{array}$ & $\begin{array}{l}\text { Aphaenops- } \\
\text { Arten }\end{array}$ \\
\hline Temperaturamplitude $\left({ }^{\circ} \mathrm{C}\right)$ & $\begin{array}{l}6,8 / 9,0 \\
6,0 / 9,0 \\
7,2 / 9,0 \\
\end{array}$ & $\begin{array}{l}6,8 / 9,0 \\
7,2 / 9,0\end{array}$ & $\begin{array}{l}8,4 / 11,0 \\
9,0 / 12,0\end{array}$ & $\begin{array}{l}6,2 / 9,0 \\
6,8 / 9,3\end{array}$ \\
\hline Anzahl der untersuchten Tiere & 19 & 7 & 25 & 23 \\
\hline Anzahl der rhytmischen Tiere & 15 & 6 & 19 & 19 \\
\hline $\begin{array}{l}\text { Aktivitätsmaximum zu Beginn der } \\
\text { Warm-und Kaltphase }\end{array}$ & 13 & 6 & 18 & 15 \\
\hline $\begin{array}{l}\text { Aktivitätsmaximum zu Beginn } \\
\text { der Warmphase }\end{array}$ & 2 & - & 1 & 4 \\
\hline $\begin{array}{l}\text { In Warm- und Kaltphase annähernd } \\
\text { gleich häufgaktiv }\end{array}$ & 6 & 3 & 16 & 9 \\
\hline In der Warmphase häufiger aktiv & 9 & 3 & 1 & 10) \\
\hline In der Kaltphase häufiger aktiv & - & - & 2 & - \\
\hline
\end{tabular}

Tab. 4. Das Verhalten im 12/12- bzw. 24/24-Std-Temperaturwechsel. 

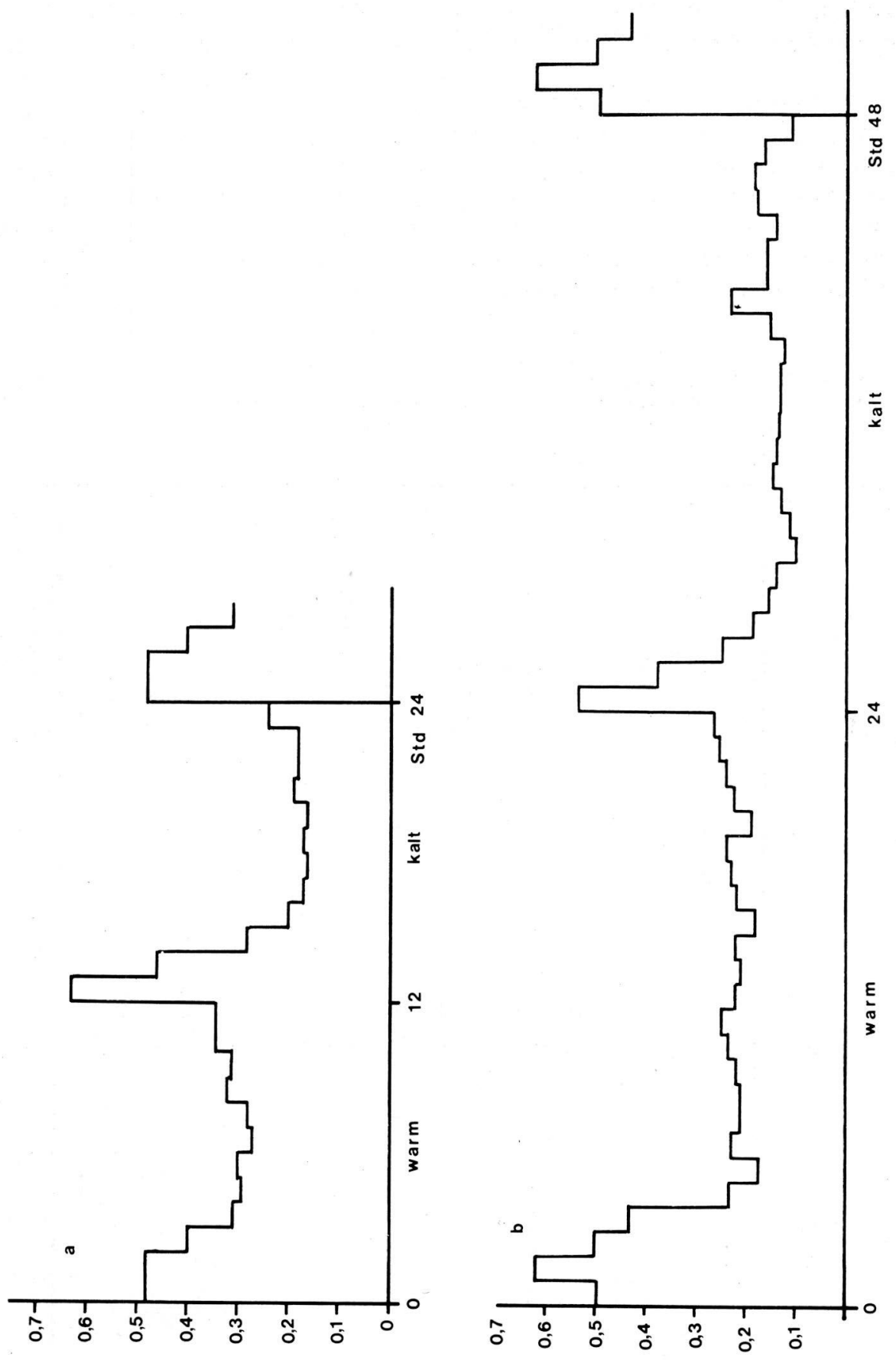

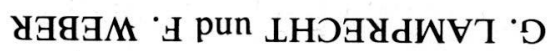



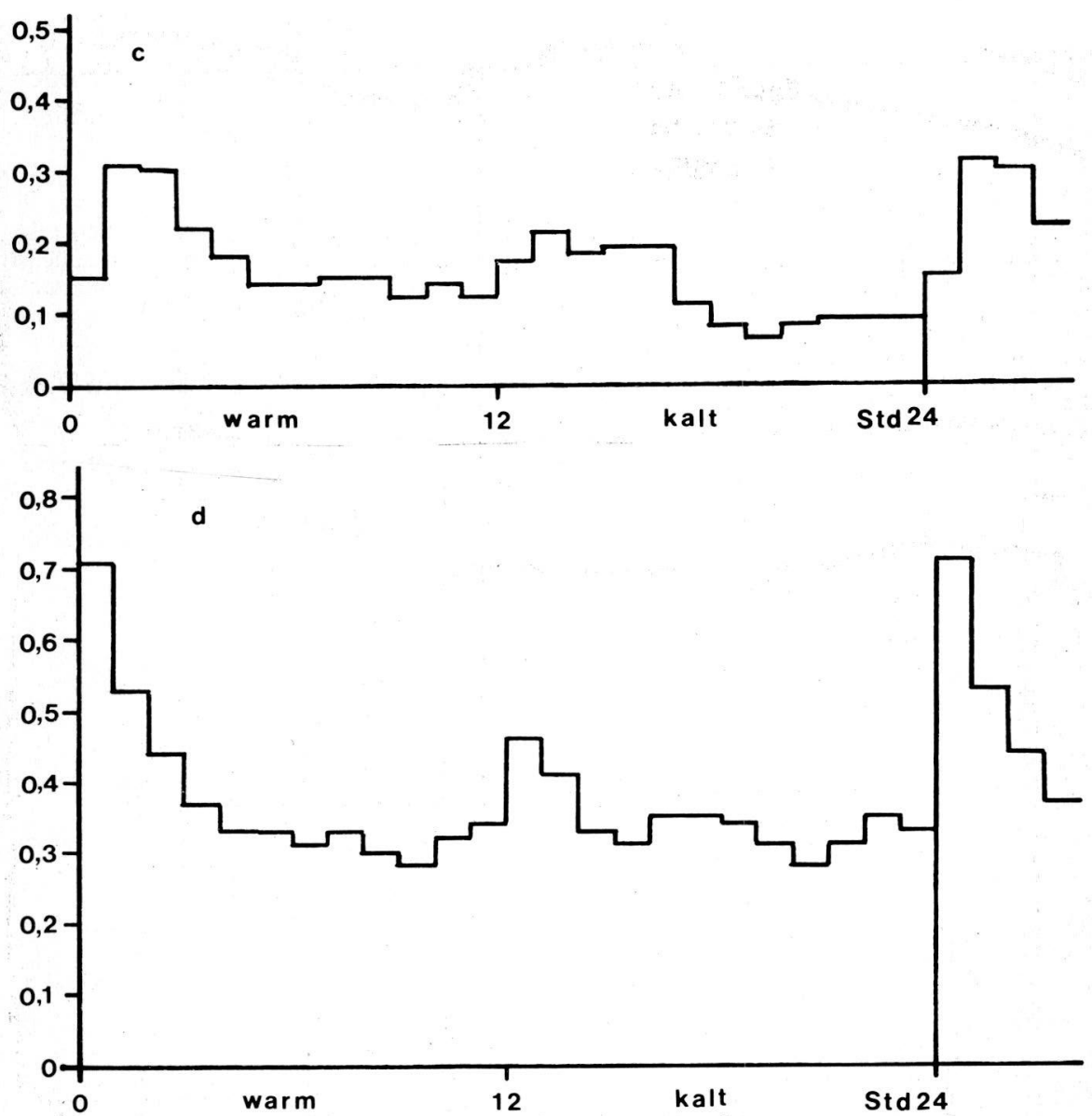

Abb. 10a - d - Die mittlere relative Schubdichte (Ordinate) in perioden-induzierenden TW (vgl. Tab. 4). a: 15 Aphaenops, 12/12 Std-TW. b: 19 Aphaenops, 24/24 Std-TW. c: 6 Geotrechus orpheus 12/12 Std-TW. d: 19 Speonomus diecki, 12/12 Std-TW.

1a, b, 10a-d, Tab. 4), und zwar durch einen Differentialeffekt (beim Wechsel von kalt nach warm und meist auch von warm nach kalt werden Aktivitätsschübe induziert) und bei Aphaenops und Geotrechus zusätzlich durch einen Proportionaleffekt (mehr als die Hälfte der untersuchten Tiere ist in der Warmphase häufiger aktiv als in der Kaltphase, Tab. 4). Aphaenops reagiert auf einen 24/24 Std-TW in gleicher Weise wie auf einen 12/12 Std-TW (Abb. 10b, Tab. 4). Die Tiere sind also nicht in besonderer Weise an tageszeitliche Veränderungen der Temperatur angepasst.

Temperaturamplituden von 1,8 bis $3,0^{\circ} \mathrm{C}$ induzieren bei fast allen Tieren eine deutliche Periodik (Tab. 4). Aber bereits bei einer Amplitude von $1,5^{\circ} \mathrm{C}$ sind 
etwa die Hälfte der Tiere aperiodisch aktiv und die übrigen werden nur noch zu einer schwachen Rhythmik induziert (Versuch: 16 Aphaenops-Individuen, 19 Tage in einem $12 / 12$ Std-TW von $7,1 \mathrm{zu} 8,6^{\circ} \mathrm{C}$ ).

Um dic Induktionswirkung im Gruppenvergleich graphisch darzustellen, wurde die «mittlere relative Schubdichte» errechnet (Abb. 10). Die "relative Schubdichte» in einer bestimmten Stunde ist 1. wenn alle 6 Messintervalle zu einem Aktivitätsschub gehören; sie ist 0,5, wenn 3 Messintervalle лu Aktivitätsschüben gehören etc. Durch Mittelung der «relativen Schubdichten» korrespóndierender Stunden ergibt sich die «mittlere relative Schubdichte».

Um den Proportionaleffekt der Temperatur auf die mittlere Schub- und Pausenlänge genau zu untersuchen, wurden 12 Aphaenops-Tiere folgendem Temperaturwechsel ausgesetzt: 7 Tage $9,5^{\circ} \mathrm{C} \rightarrow 7$ Tage $6^{\circ} \mathrm{C} \rightarrow 7$ Tage $9,5^{\circ} \mathrm{C}$. Von der Temperatursenkung bleibt $\overline{\mathrm{A}}$ im Mittel fast unbeeeiflusst $\left(\mathrm{Q}_{10}=\right.$ $0,89)$, während $\bar{R}$ im Mittel länger wird $\left(Q_{10}=0,35\right)$. Nach dem erneuten
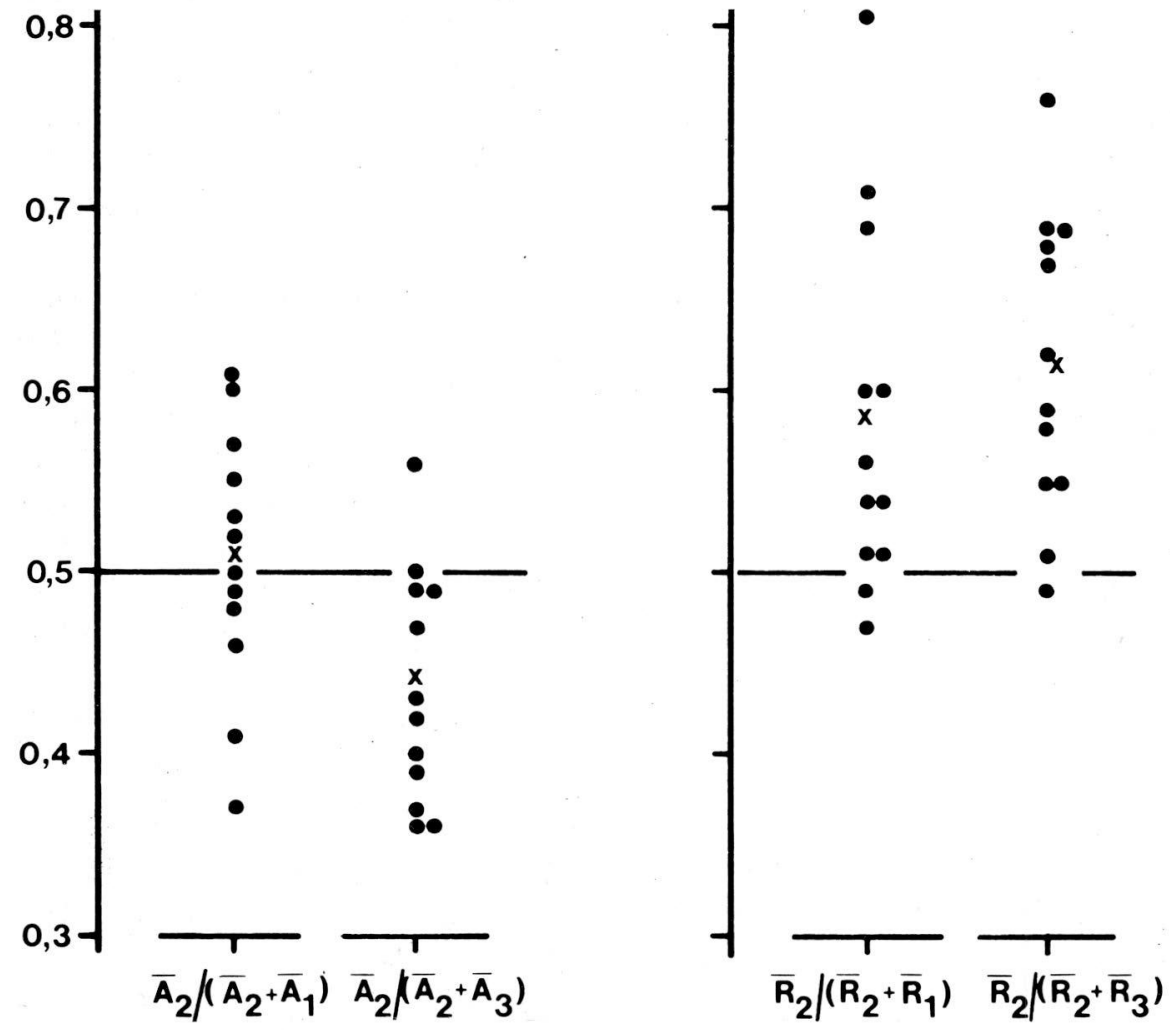

Abb. 11 - Die Temperaturabhängigkeit der mittleren Schublängen $(\overline{\mathrm{A}})$ und Pausenlängen $(\overline{\mathrm{R}})$ bei

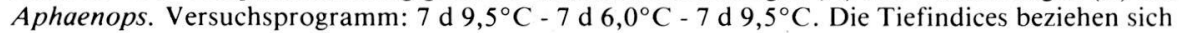
auf die 3 Versuchsabschnitte.

- Einzeltierwerte, $\mathbf{x}$ gemittelte Werte. $\bar{A}$ ist weniger temperaturabhängig als $\bar{R}$. 
Temperaturanstieg im 3. Versuchsabschnitt wird $\overline{\mathrm{A}}$ im Mittel länger $\left(\mathrm{Q}_{10}=\right.$ $2,24)$ und $\bar{R}$ wieder kürzer $\left(Q_{10}=0,28\right)$ (Abb. 11). Demnach scheint $\bar{A}$ im Mittel weniger temperaturabhängig zu sein als $\overline{\mathrm{R}}$. - Im übrigen entspricht die gegensinnige Temperaturabhängigkeit der (schwachen) negativen Korrelation zwischen $\overline{\mathrm{A}}$ und $\overline{\mathrm{R}}$.

\section{DISKUSSION}

\subsection{Die Evolution stochastischer Aktivitäts-Muster}

Zufallsgesteuerte Aktivitätsmuster mit zeitinvarianten oder regelhaft zeitabhängigen Übergangswahrscheinlichkeiten wurden bisher bei Winkerkrabben (Lehmann, Neumann und Kaiser 1974), beim sibirischen Streifenhörnchen (Eutamias sibiricus), bei der Gopherschildkröte (Gopherus polyphemus), bei der Erdmaus (Microtus agrestis), bei menschlichen Säuglingen (Lehmann, 1976) sowie bei Schaben (Blaberus fuscus) nach Bilobektomie (Lukat, unveröffentlicht) beschrieben. Auch von anderen Verhaltenssequenzen bei Evertebraten und Vertebraten ist Zufallssteuerung bekannt (Kavanau, 1963; Delius, 1969; Markl u. Hauff, 1973; Kloot u. Morse, 1975).

Möglicherweise stellt die Aktivitätskontrolle durch endogene zentralnervöse «Zufallsgeneratoren» ein weit verbreitetes Phänomen dar. Bei den meisten epigäisch aktiven Arten wird dieser Mechanismus offenbar durch die circadiane Uhr überlagert und ist erst dann fassbar, wenn die Uhr ausgeschaltet wird (z.B. bei Schaben nach Bilobektomie). Wenn in der regressiven Evolution der Höhlentiere das circadiane Zeitmess-System degeneriert, scheinen die endogenen «Zufallsgeneratoren» bei der Kontrolle der Aktivität das Übergewicht zu erlangen. Diese Hypothese impliziert, dass im Zuge der Evolution unter Höhlenbedingungen ein spezieller aktivitätskontrollierender Mechanismus nicht entstand.

Bei Höhlentieren können heute 5 verschiedene Formen der Aktivitätskontrolle unterschieden werden. Der Anteil der periodischen Komponenten in den Aktivitätsmustern nimmt in diesem Schema sukzessiv ab.

1.) Troglophile Arten mit einer intakten Circadian-Uhr, die die Aktivität sowohl im DD als auch LL und LD kontrolliert. Beispiele: die Laufkäfer Laemostenus terricola und oblongus (Lamprecht u. Weber, 1975, 1977).

2.) Troglobionte Arten mit einer intakten Circadian-Uhr, die die Aktivität im wesentlichen aber nur im LL und LD kontrolliert, während im DD stochastische Aktivitätsmuster vorherrschen. Beispiel: der Höhlenlaufkäfer Laemostenus navarricus (Lamprecht $u$. Weber, 1975, 1977). Diese Art ist auch in mor- 
phologischen Merkmalen weiter evoluiert als L. terricola und oblongus.

3.) Höhlentiere ohne selbsterregte Circadian-Rhythmik, aber mit der Fähigkeit, nach einem Übergang von einem rhythmusinduzierenden LD zu DD oder LL gedämpft nachzuschwingen. Wenn die Nachschwingungen ausgeklungen sind, ist die Aktivität stochastisch verteilt. Beispiel: die blinde Höhlenform des Fisches Astyanax mexicanus (Erckens u. Weber, 1976).

4.) Blinde Arten ohne selbsterlgte Circadian-Rhythmik und ohne Nachschwingungen der Aktivität nach einem Übergang von LD zu Konstantbedingungen. LD induzieren noch eine Periodik. Beispiel: die blinden cavernicolen Amphipoden der Gattung Niphargus (Ginet, 1960; Günzler, 1964). Ob unter Konstantbedingungen die Aktivität nach einfachen stochastischen Regelhaftigkeiten verteilt wird, wurde nicht untersucht.

5.) Blinde Arten, die nicht mehr auf LD reagieren. Die Aktivität ist bei Temperaturk onstanz stochastisch verteilt. Temperaturwechsel induzieren eine Aktivitätsperiodik. Beispiele: Arten der Gattungen Aphaenops und Geotrechus.

Die an anderen Höhlentieren durchgeführten Untersuchungen sind zu fragmentarisch, als dass eine Einordnung in dieses Schema oder die Einfügung weiterer Zwischenstufen möglich wäre. Beim Höhlenkrebs Orconectes pellucidus beobachteten Park, Roberts u. Harris (1941) keinen Aktivitätsrhythmus, während Jegla u. Poulson (1968) bei einigen Individuen dieser Art im DD signifikante Periodizitäten der Schwimmaktivität und des $0_{2}$-Verbrauchs gefunden haben wollen. Das LD- und LL-Verhalten der Art wurde noch nicht genauer untersucht.

Nach Günzler (1964) und Mead u. Gilhodes (1974) weisen die Aktivitätsmuster der Höhlenamphipode Niphargus puteanus bzw. der Höhlendiplopode Blaniulus lichtensteini ein breites Spektrum von Schwingungen auf, das auch den ultradianen und infradianen Bereich umfasst. Da die Perioden jeweils nur über kürzere Abschnitte nachweisbar sind, vermuten wir, dass in beiden Fällęn die Aktivität stochastisch verteilt ist und die beobachteten stark gestörten «Perioden» lediglich zufällige Folgen ungefähr gleich langer $(A+R)$-Werte darstellen.

\subsection{Adaptive Reaktionen der Zufallssteuerung}

Durch eine Zufallssteuerung der Aktivität kann - auch im Falle konstanter, zeitinvarianter Übergangswahrscheinlichkeiten - zuverlässig eine den speziellen Umweltbedingungen angepasste mittlere Schub- bzw. Pausenlänge konstant gehalten werden. Ist der zugrunde liegende (noch hypothetische) zentralnervöse Steuerungsmechanismus zu adaptiven Reaktionen befähigt? Adapti- 
ves Reagieren ist für die endogene Circadianuhr vielfach bewiesen (z.B. Synchronisierbarkeit, Temperaturkompensation der Periode). Auch die zufallsgesteuerten Aktivitätsmuster der Winkerkrabben passen sich den Gezeiten und Licht-Dunkel-Wechseln an (Lehmann, Neumann u. Kaiser, 1974). -Bei Höhlentieren könnte die Fähigkeit zu adaptiven Reaktionen während der Evolution unter Konstantbedingungen eingeschränkt worden sein. Insofern überrascht es nicht, dass der Mechanismus der Zufallssteuerung bei den hier untersuchten Höhlentieren nur noch schwache adaptive Reaktionen erkennen lässt. Aphaenops und Geotrechus reagieren nicht mehr auf Änderungen der Belichtung. Im Verlauf der dreiwöchigen Versuche (d.h. während einer relativ langen Hungerzeit) verändern sich - mit Ausnahme von $\bar{R}$ bei Speonomus - die mittleren Schub- und Pausenlängen nicht regelhaft. - Auf ein reliktäres adaptives Reaktionsvermögen deuten andererseits bei Aphaenops, Geotrechus und Speonomus der Differentialeffekt an beiden Phasengrenzen eines Temperaturzyklus (kalt $\rightarrow$ warm und warm $\rightarrow$ kalt) (Abb. 10), bei Speonomus im Temperaturwechsel das Fehlen eines Proportionaleffektes (Abb. 10d) sowie bei Aphaenops die geringe Temperaturabhängigkeit der mittleren Schublänge (Abb. 11). Auch die schwache negative Korrelation zwischen den Veränderungen von $\overline{\mathrm{A}}$ und $\overline{\mathrm{R}}$ bei Aphaenops (Abb. 9a) kann als Relikt adaptiven Reaktionsvermögens angesehen werden.

\subsection{Kriterien stochastischer Sequenzen}

Zufallsgesteuerte Aktivitätsmuster liegen dann vor, wenn die Längen der aufeinanderfolgenden Aktivitätsschübe und Ruhepausen nicht miteinander korreliert sind. Dabei können die die Sequenz determinierenden Wahrscheinlichkeitsprozesse zeitabhängig oder zeitunabhängig (zeitinvariant) sein. Zufallsgesteuert sind sowohl die Aphaenops-Aktogramme mit exponentiellen Häufigkeitsverteilungen (zeitinvariante Übergangswahrscheinlichkeiten) als auch die Geotrechus- und Speonomus-Aktogramme, deren Häufigkeitsverteilungen ein Maximum im Bereich mittlerer Klassen aufweisen (zeitabhängige Übergangswahrscheinlichkeiten). Je ausgeprägter die Zeitabhängigkeit wird, um so kleiner wird die Streuung der Schub- bzw. Pausenlängen sein und um so häufiger und deutlicher werden im Aktogramm «rhythmische» Folgen auftreten (vgl. Kaiser u. Lehmann, 1975). Da jedoch in einem solchen System zufällige Phasenverschiebungen von erheblichem Ausmass auftreten können, ist es unwahrscheinlich, dass circadianperiodische Aktivitätsmuster auf diese Weise gesteuert werden. Wahrscheinlicher ist es, dass periodische Muster von einem Oszillator (einer «Uhr») kontrolliert werden, der durch kompensatorische Veränderungen der Länge der Aktivitäts- und Ruhephasen die Einhaltung einer mittleren Periodenlänge garantiert. 
Wir glauben nicht, dass zwischen der Aktivitätssteuerung bei, Aphaenops (zeitinvariante Übergangswahrscheinlichkeiten) und Geotrechus bzw. Speonomus (häufig zeitabhängige Wahrscheinlichkeiten) ein grundsätzlicher Unterschied besteht. Eine Veränderung der Übergangswahrscheinlichkeit von Zeitabhängigkeit nach Zeitunabhängigkeit haben wir auch bei ein- und demselben Tier innerhalb eines Versuchs beobachten können (vgl. z.B. Abb. 7).

Es kann nicht übersehen werden, dass eine nicht unbeträchtliche Anzahl von Verteilungen, insbesondere solche der Ruhepausen, sich nicht einer der drei getesteten Funktionen zuordnen lassen (Tab. 2). Nach welchen Regelhaftigkeiten sich die Übergangswahrscheinlichkeiten in diesen Fällen verändern, bleibt zunächst unbekannt. Allerdings sind die gemittelten empirischen Übergangswahrscheinlichkeiten zum Teil ebenfalls zeitinvariant oder nehmen stetig mit der Zeit zu (Abb. 8).

\section{DANKSAGUNG}

Für freundliche Unterstützung und Hilfe beim Fang der Untersuchungstiere danken wir Herrn Dr. C. Juberthie und Herrn M. Bouillon, Laboratoire Souterrain, Moulis (Ariège, Frankreich).

\section{ZUSAMMENFASSUNG}

Bei konstanter Temperatur sind die troglobionten Höhlenkäfer Aphaenops cerberus und A. pluto, Geotrechus orpheus und Speonomus diecki aperiodisch aktiv. Durch eine Periodogrammanalyse können in den Aktogrammen weder circadiane, noch ultradiane oder infradiane periodische Komponenten nachgewiesen werden. Auch bedeutsame Korrelationen zwischen den Längen aufeinander folgender Aktivitätsschübe und Ruhepausen sind nicht vorhanden..Die Aktivitätsmuster sind demnach stochastisch strukturiert. - In den Fällen, in denen das Aktivitäts- bzw. Ruheverhalten während des ganzen Versuches (16 - 20 Tage) oder wenigstens während der halben Versuchszeit konstant ist, werden aus den Häufigkeitsverteilungen der Schub- bzw. Pausenlängen Übergangswahrscheinlichkeiten errechnet. Die Übergangswahrscheinlichkeiten ist zeitinvariant, wenn sich die beobachtete Verteilung nicht von der Exponentialfunktion $y=a \cdot e^{-f \cdot x}$ unterscheiden läßt; sie wird mit der Dauer eines Schubes bzw. einer Pause kontinuierlich größer, wenn sich die beobachtete Verteilung nicht von einer Poisson- oder Normalverteilung unterscheiden lässt. $-74 \%$ der untersuchten Häufigkeitsverteilungen der Aktivitätsschublängen und 57\% der Verteilungen der Ruhepausenlängen liessen sich durch eine der genannten theoretischen Funktionen beschreiben. -Aphaenops und Geotrechus reagieren nicht auf 12/12 Std Licht-Dunkel-Wechsel. Hingegen induzieren Temperaturzyklen eine Aktivitätsperiodik. Bei Aphaenops ist die mittlere Aktivitätsschublänge weniger temperaturabhängig als die mittlere Ruhepausenlänge. Ausserdem ist bei dieser Art die mittlere Schublänge schwach negativ mit der mittleren Pausenlänge korreliert. - Es werden die Evolution und die adaptiven Reaktionen des Mechanismus der Zufalls-steuerung bei Höhlentieren diskutiert.

\section{LITERATUR}

BATSCHELET E., - Statistical rhythm evaluation. In: Biorhythms and Human Reproduction (eds. M. Ferin, F. Halberg, R. M. Richard, R. L. van de Wiele). S. 25-35. New York, London, Sidney, Toronto 1974.

DELIUS J.D., - A stochastic analysis of the maintenance behaviour of Skylarks. Behaviour 33, 137-178 (1969).

ERCKENS W. \& F. WEBER., - Rudiments of an ability for time measurement in the cavernico- 


\section{AKTIVITÄTSMUSTER BEI TROGLOBIONTEN KÄFERN}

lous fish Anoptichthys jordani Hubbs and Innes (Pisces, Characidae). Experientia 32, 1297-1299 (1976).

GINET R., - Écologie, éthologie et biologie de Niphargus. Ann. Spéléol. 15, 127-379 (1960).

GLACON S., - Recherches sur la biologie des Coléoptères cavernicoles troglobies. Premier Congrès Intern. Spéléol. Comm. III, 39-45 (1953).

GÜNZLER E., - Über den Verlust der endogenen Tagesrhythmik bei dem Höhlenkrebs Niphargus puteanus puteanus (KOCH). Biol. Zentralbl. 83, 677-694 (1964).

JEGLA T.C. \& T.L. POULSON., - Evidence of circadian rhythms in a cave crayfish. J. exp. Zool. 168, 273-282 (1968).

JUBERTHIE C., - Relations entre le climat, le microclimat et les Aphaenops cerberus dans la grotte de Sainte-Cathrine (Ariège). Ann. Spéléol. 24, 75-104 (1969).

KAISER H \& U. LEHMANN., - Tidal and spontaneous activity patterns in Fiddler Crabs II. Stochastic models and simulations. J. comp. Physiol. 96, 1-26 (1975).

KAVANAU J.L., - Continuous automatic monitoring of the activities of small captive animals. Ecology 44, 95-110 (1963).

KLOOT W. von der \& M.J. MORSE., - A stochastic analysis of the display behavior of the redbreasted merganser (Mergus serrator). Behaviour 54, 181-216 (1975).

LAMPRECHT G. \& F. WEBER., Eine neue Methode zur Bestimmung von Periodenlängen rhythmisch ablaufender physiologischer Prozesse. Pflügers Arch. 315, 262-272 (1970).

LAMPRECHT G. \& F. WEBER., - Die Circadian-Rhythmik von drei unterschiedlich weit an ein Leben unter Höhlenbedingungen adaptierten Laemostenus-Arten (Col. Carabidae). Ann. Spéléol: 30, 471-482 (1975).

LAMPRECHT G. \& F. WEBER., - Die Lichtempfindlichkeit der circadianen Rhythmik dreier Höhlenkäfer der Gattung Laemostenus. J. Insect. Physiol. 23, 445-452 (1977).

LEHMANN U., - Stochastic principles in the temporal control of activity behaviour. Int. J. Chronobiol, 4, 223-266 (1976).

LEHMANN U., D. NEUMANN \& H. KAISER., - Gezeitenrhythmische und spontane Aktivitätsmuster von Winkerkrabben. I. Ein neuer Ansatz zur quantitativen Analyse von Lòkomotionsrhythmen, J. comp. Physiol. 91, 181-221 (1974).

LUKAT R. \& F. WEBER., - Die Struktur der arhythmischen Aktivität gehirnoperierter Schaben (Blaberus fuscus). In preparation.

MARKL H. \& J. HAUFF., - Die Schwellenkurve des durch Vibration aus-gelösten Fluchttauchens von Mückenlarven. Naturwissenschaften 60, 432-433 (1973).

MEAD M. \& J.C. GILHODES., Organisation temporelle de l'activité locomotrice chez un animal cavernicole Blaniulus lichtensteini Bröl. (Diplopoda). J. comp. Physiol. 90, 47-52 (1974).

PARK O., T.W. ROBERTS \& S.J. HARRIS., - Preliminary analysis of activity of the cave crayfish, Cambarus pellucidus. Amer. Nat. 75, 154-171 (1941).

SACHS L., - Angewandte Statistik. 4. Aufl. Berlin, Heidelberg, New York 1974.

VANDEL A., - Biospeleology. Oxford, London, Paris 1965.

VERVEEN A.A., - An Introduction to the Use of Time Series Analysis in Physiology. In: Reichardt, W., Rendiconti della Scuola Internazionale di Fisica «Enrico Fermi», XLIII Corso. Milano 1969. 